\title{
INFINITE FAMILIES OF EQUIVARIANTLY FORMAL TORIC ORBIFOLDS
}

\author{
ANTHONY BAHRI, SOUMEN SARKAR, AND JONGBAEK SONG
}

\begin{abstract}
The simplicial wedge construction on simplicial complexes and simple polytopes has been used by a variety of authors to study toric and related spaces, including non-singular toric varieties, toric manifolds, intersections of quadrics and more generally, polyhedral products. In this paper we extend the analysis to include toric orbifolds. Our main results yield infinite families of toric orbifolds, derived from a given one, whose integral cohomology is free of torsion and is concentrated in even degrees, a property which might be termed integrally equivariantly formal. In all cases, it is possible to give a description of the cohomology ring and to relate it to the cohomology of the original orbifold.
\end{abstract}

\section{INTRODUCTION}

Compact smooth toric varieties $X$, and their topological counterparts, toric manifold $₫$, have their integral cohomology torsion free and concentrated in even degrees. Consequently, the action of the compact $n$-dimensional torus $T=\left(S^{1}\right)^{n}$ on $X$, allows for a satisfying description of the integral cohomology ring arising from the collapsing Serre spectral sequence of the canonical fibration

$$
X \stackrel{\iota}{\hookrightarrow} E T \times_{T} X \stackrel{\pi}{\rightarrow} B T \cong\left(\mathbb{C} P^{\infty}\right)^{n} .
$$

Franz and Puppe note in [FP07, Theorem 1.1], that for compact smooth toric varieties, or more generally, a finite $T$-CW complexes, this is equivalent to the map $\iota^{*}$ inducing an isomorphism

$$
H^{*}\left(E T \times_{T} X\right) \otimes_{H^{*}(B T)} \mathbb{Z} \longrightarrow H^{*}(X) .
$$

The term integrally equivariantly formal suggests itself for $X$, by analogy with the case of rational coefficients, see for instance [GKM98. In this smooth setting, (1.2) leads to a description of the ring $H^{*}(X)$ as a quotient of a Stanley-Reisner ring by a linear ideal.

The situation for singular toric spaces is not so nice; examples with non-vanishing cohomology in odd degrees abound, Fis92, CLS11, Chapter 12]. In the case of orbifolds however, the results of BNSS establish tractable sufficient conditions ensuring that the integral cohomology is torsion free and concentrated in even degree. So, for such spaces an integral equivariant formality holds and the ring structure of the cohomology can be identified explicitly, in a manner entirely analogous to

2010 Mathematics Subject Classification. 13F55, 14M25, 52B11, 57R18, 55N91.

Key words and phrases. toric variety, orbifold, singular cohomology, equivariantly formal, simplicial wedge, J-construction.

${ }^{1}$ Though Davis and Januszkiewicz [DJ91] introduced the name toric manifolds, in recent literature they are sometimes called quasitoric manifolds. 
the smooth case described above; the Stanley-Reisner ring is replaced by a ring of certain piecewise polynomials or weighted Stanley-Reisner rings, see [BFR09, Proposition 2.2] and [BSS17, Theorem 5.3].

In this sequel to BNSS, we extend the program to a class of infinite families of toric orbifolds, derived from a given one by a combinatorial construction, known variously as: the simplicial wedge construction [PB80, Ewa86, the doubling construction LdM89, and the J-construction BBCG, BBCG15].

The construction associates to a sequence of positive integers $J=\left(j_{1}, j_{2}, \ldots, j_{m}\right)$ and an $(n-1)$-dimensional simplicial complex $K$ on $m$ vertices, a new simplicial complex $K(J)$ on $d(J)=j_{1}+j_{2}+\cdots+j_{m}$ vertices, of dimension $d(J)-m+n-1$. Equally well, it associates to a simple polytope $Q$ of dimension $n$ with $m$ facets, a new simple polytope $Q(J)$ of dimension $d(J)-m+n$ having $d(J)$ facets. (Notice that $m-n=d(J)-(d(J)-m+n)$.)

As outlined in Section 2 below, a $2 n$-dimensional toric orbifold $X(Q, \lambda)$ is specified by an $\mathcal{R}$-characteristic pair $(Q, \lambda)$ where $Q$ is an $n$-dimensional simple polytope and $\lambda: \mathcal{F}(Q) \rightarrow \mathbb{Z}^{n}$ is a function from the set of facets of $Q$ which satisfies certain conditions.

Section 4 describes the way in which each sequence $J$, determines from $X(Q, \lambda)$ a new $2(d(J)-m+n)$-dimensional toric orbifold $X_{(J)}:=X\left(Q_{(J)}, \lambda_{(J)}\right)$. Our goal here is two-fold:

(1) To confirm that if $X(Q, \lambda)$ satisfies the sufficiency conditions of BNSS, Theorem 1.1], which ensure that the integral cohomology is torsion free and concentrated in even degree, then as $J$ varies, the spaces $X\left(Q_{(J)}, \lambda_{(J)}\right)$ yield an infinite family of similarly integrally equivariantly formal orbifolds.

(2) To relate the integral cohomology ring of $X_{(J)}$ to that of $X$.

The elementary theory of toric orbifolds is reviewed in Section 2, drawing from the expositions to be found in DJ91, PS10 and BSS17. The emphasis is on tracking the finite isotropy groups and the singularities resulting from the failure of the $\mathcal{R}$-characteristic function $\lambda$ to satisfy the regularity condition which ensures the smoothness of a toric space.

The orbifold analogue of a CW-complex, called a q-CW complex, developed rationally in the toric space setting by Poddar and Sarkar [PS10] and integrally in BNSS, is reviewed in Section 3. The $\mathbf{q}-\mathrm{CW}$ complex structure is connected to the underlying combinatorics by the notion of a retraction sequence for a simple polytope, introduced in BSS17. The outcome of these observations allows for an iterated construction of the toric orbifold via a sequence of cofibrations which keep track of the isotropy, and hence singularities, as they arise. A condition involving the divisibility of all the orders of the isotropy groups, which emerge from particular retraction sequences, proves sufficient to establish the integral equivariant formality of the toric orbifold. The cornerstone of several of the results presented here, is the next theorem.

Theorem 3.7 [BNSS, Theorem 4.6] Let $X:=X(Q, \lambda)$ be a toric orbifold. If for each prime number $p$ there is a retraction sequence $\left\{\left(B_{j}, E_{j}, b_{j}\right)\right\}_{j=1}^{\ell}$ such that $\operatorname{gcd}\left\{p,\left|G_{E_{j}}\left(b_{j}\right)\right|\right\}=1$ for all $j$, then $H_{*}(X ; \mathbb{Z})$ has no torsion and $H_{\text {odd }}(X ; \mathbb{Z})$ is trivial.

In Section 4 various formulations of the simplicial wedge construction are introduced on both simplicial complexes and simple polytopes. This is followed by a 
description of the transition of $\mathcal{R}$-characteristic pairs

$$
X(Q, \lambda) \rightsquigarrow X\left(Q_{(J)}, \lambda_{(J)}\right)
$$

for each sequence $J=\left(j_{1}, j_{2}, \ldots, j_{m}\right) \in \mathbb{N}^{m}$. Included here is the verification, in Lemma 4.3. that if the $\mathcal{R}$-characteristic map $\lambda$ satisfies the orbifold condition (2.1), then $\lambda_{(J)}$ does too. This is a modification of the argument for the smooth case, BBCG15, Theorem 3.2]. The construction of $Q_{(J)}$ from $Q$ is an iterative process involving a sequence of "doubling" operations. Though it is well known that the result is independent of the particular sequence chosen, we include here Proposition 4.6 and the details of a proof for completeness.

Section 5 is devoted to the homology groups of the toric orbifold $X_{(J)}:=$ $X\left(Q_{(J)}, \lambda_{(J)}\right)$; the main theorem is the following.

Theorem 5.5 Let $X:=X(Q, \lambda)$ be a toric orbifold satisfying the assumption of Theorem 3.6. Then, $H_{*}\left(X_{(J)} ; \mathbb{Z}\right)$ is torsion free and $H_{\text {odd }}\left(X_{(J)} ; \mathbb{Z}\right)$ vanishes for arbitrary $J=\left(j_{1}, j_{2}, \ldots, j_{m}\right) \in \mathbb{N}^{m}$.

The paper concludes with a discussion of the integral cohomology ring of the toric orbifolds $X_{(J)}$. When the toric orbifold $X(Q, \lambda)$ satisfies the hypothesis of Theorem 3.7, then Theorem 6.5 characterizes the cohomology of $X_{(J)}$ as the quotient of a certain weighted Stanley-Reisner ring $w \mathcal{S R}\left[Q_{(J)}, \lambda_{(J)}\right]$ by a linear ideal which depends on the $\mathcal{R}$-characteristic map $\lambda$. The final result relates the ring $w \mathcal{S} \mathcal{R}\left[Q_{(J)}, \lambda_{(J)}\right]$ to the ring $w \mathcal{S} \mathcal{R}[Q, \lambda]$.

Acknowledgments. This work was supported in part by grants 210386 and 426160 from Simons Foundation. The third author has been supported by the POSCO Science Fellowship of POSCO TJ Park Foundation.

\section{TORIC ORBIFOLDS}

In this section, we review the basic theory of toric orbifolds DJ91, PS10, constructed from a combinatorial information called an $\mathcal{R}$-characteristic pair [BSS17. Given an $n$-dimensional simple convex polytope $Q$, let $V(Q)=\left\{v_{1}, \ldots, v_{\ell}\right\}$ be the set of vertices and $\mathcal{F}(Q)=\left\{F_{1}, \ldots, F_{m}\right\}$ the set consisting of codimension 1 faces called facets of $Q$.

Definition 2.1. A pair $(Q, \lambda)$ consisting of an $n$-dimensional simple polytope $Q$ and a function $\lambda: \mathcal{F}(Q) \rightarrow \mathbb{Z}^{n}$, is called an $\mathcal{R}$-characteristic pair if the following condition is satisfied:

$$
\left\{\lambda\left(F_{i_{1}}\right), \ldots, \lambda\left(F_{i_{k}}\right)\right\} \text { is a linearly independent set, whenever } \bigcap_{j=1}^{k} F_{i_{j}} \neq \emptyset .
$$

In this case, we call $\lambda$ an $\mathcal{R}$-characteristic function on $Q$.

An $\mathcal{R}$-characteristic function is often represented by an $n \times m$ matrix whose $i$-th column vector is the transpose of $\lambda\left(F_{i}\right)$ for $i=1, \ldots, m$. We call this matrix the characteristic matrix associated to $\lambda$.

Given an $(n-k)$-dimensional face $E=F_{i_{1}} \cap \cdots \cap F_{i_{k}}$ of $Q$ for $k \geq 1$, let $M(E)$ be the $\mathbb{Z}$-submodule of $\mathbb{Z}^{n}$ generated by the set $\left\{\lambda\left(F_{i_{1}}\right), \ldots, \lambda\left(F_{i_{k}}\right)\right\}$. Then, $M(E)$ induces a free $\mathbb{Z}$-submodule $\left(M(E) \otimes_{\mathbb{Z}} \mathbb{R}\right) \cap \mathbb{Z}^{n}$ of rank $k$ in $\mathbb{Z}^{n}$. Hence, we can define a natural projection

$$
\rho_{E}: \mathbb{Z}^{n} \rightarrow \mathbb{Z}^{n-k} \cong \mathbb{Z}^{n} /\left(\left(M(E) \otimes_{\mathbb{Z}} \mathbb{R}\right) \cap \mathbb{Z}^{n}\right) .
$$


Next, set

$$
\mathcal{F}(E):=\left\{E \cap F_{j} \mid F_{j} \notin\left\{F_{i_{1}}, \ldots, F_{i_{k}}\right\} \text { and } F_{j} \cap E \neq \emptyset\right\} .
$$

Now, we define a function

$$
\lambda_{E}: \mathcal{F}(E) \rightarrow \mathbb{Z}^{n-k} \quad \text { by } \quad \lambda_{E}\left(E \cap F_{j}\right)=\operatorname{prim}\left(\left(\rho_{E} \circ \lambda\right)\left(F_{j}\right)\right),
$$

where $\operatorname{prim}\left(\left(\rho_{E} \circ \lambda\right)\left(F_{j}\right)\right)$ denotes the primitive vector of $\left(\rho_{E} \circ \lambda\right)\left(F_{j}\right)$. One can check that the pair $\left(E, \lambda_{E}\right)$ is an $\mathcal{R}$-characteristic pair. We also associate $M(E)$ with a $k$-dimensional subtorus $T_{E}$ of standard $n$-dimensional real torus $T^{n}$ generated by the images of $\lambda\left(F_{i_{1}}\right), \ldots, \lambda\left(F_{i_{k}}\right)$ under the map $M(E) \hookrightarrow \mathbb{Z}^{n} \stackrel{\exp }{\longrightarrow} T^{n}$.

An $\mathcal{R}$-characteristic pair $(Q, \lambda)$ determines a $2 n$-dimensional orbifold with an action of $n$-dimensional torus $T^{n}$. To be more precise, for each point $x \in E$, let $E(x)$ be the face of $Q$ containing $x$ in its interior. Now we consider the following quotient space

$$
X(Q, \lambda):=\left(T^{n} \times Q\right) / \sim,
$$

where

$$
(t, x) \sim(s, y) \text { if and only if } x=y \text { and } t^{-1} s \in T_{E(x)} .
$$

The space $X(Q, \lambda)$ is equipped with an action of $T^{n}$ given by the multiplication on the first factor. The orbit map is induced by the projection onto the second factor,

$$
\pi: X(Q, \lambda) \rightarrow Q
$$

defined by $[t, x]_{\sim} \mapsto x$. A detailed verification that $X(Q, \lambda)$ has an orbifold structure, including an explicit description of the orbifold charts, is contained in PS10, Section 2]. The authors also give an axiomatic description and show that it agrees with the construction above up to equivariant homeomorphism. The space $X(Q, \lambda)$ is known as a toric orbifold?.

Remark 2.2. If the collection $\left\{\lambda\left(F_{i_{1}}\right), \ldots, \lambda\left(F_{i_{k}}\right)\right\}$ in (2.1) is unimodular whenever $F_{i_{1}} \cap \cdots \cap F_{i_{k}} \neq \emptyset$, then $\lambda$ is called a characteristic function and the resulting space $X(Q, \lambda)$ is a smooth toric manifold, see [DJ91, Section 1].

Remark 2.3. An $\mathcal{R}$-characteristic pair $(Q, \lambda)$ induces another $\mathcal{R}$-characteristic pair $\left(E, \lambda_{E}\right)$ for each face $E$ of $Q$, which defines another toric orbifold $X\left(E, \lambda_{E}\right)$. On the other hand, $\pi^{-1}(E)$ is an invariant suborbifold of $X(Q, \lambda)$ with respect to the action of $T^{n}$ on $X(Q, \lambda)$. Indeed, $\pi^{-1}(E)$ is the suborbifold fixed by the subtorus $T_{E}$ of $T^{n}$. The residual torus $T^{n} / T_{E} \cong T^{\operatorname{dim} E}$ acts on the suborbifold $X\left(E, \lambda_{E}\right)$ and $\pi^{-1}(E)$. It is shown in PS10, Section 2.3] that $X\left(E, \lambda_{E}\right)$ is equivariantly homeomorphic to $\pi^{-1}(E)$. In particular, when $E$ is a 1-dimensional face of $Q$, then $\pi^{-1}(E)$ is homeomorphic to $S^{2}$.

For simplicity, we summarize notation that we introduced above as follows.

(1) $(Q, \lambda)$ is an $\mathcal{R}$-characteristic pair with $\operatorname{dim} Q=n$ and $X(Q, \lambda)$ is the associated toric orbifold of dimension $2 n$.

(2) $E=F_{i_{1}} \cap \cdots \cap F_{i_{k}}$ is an $(n-k)$-dimensional face of $Q$, for $k \geq 1$.

(3) $\lambda_{E}: \mathcal{F}(E) \rightarrow \mathbb{Z}^{n-k}$ is an $\mathcal{R}$-characteristic function induced from $(Q, \lambda)$.

(4) $X\left(E, \lambda_{E}\right)$ is the toric orbifold associated to the $\mathcal{R}$-characteristic pair $\left(E, \lambda_{E}\right)$.

\footnotetext{
${ }^{2}$ Davis and Januszkiewicz DJ91 first called them toric orbifolds. In recent literature, they are sometimes called quasitoric orbifolds.
} 


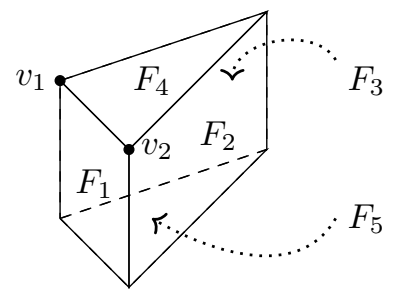

FIGURE 1.

(5) For a vertex $v$ in $E$, we denote by $\Lambda_{E, v}: \mathbb{Z}^{n-k} \rightarrow \mathbb{Z}^{n-k}$ the linear map given by the square matrix

$$
\Lambda_{E, v}=\left[\lambda_{E}\left(E \cap F_{s_{1}}\right)^{t}|\ldots| \lambda_{E}\left(E \cap F_{s_{n-k}}\right)^{t}\right],
$$

where $v=\bigcap_{a=1}^{n-k}\left(E \cap F_{s_{a}}\right)$. In particular, when $E=Q$ and $v=\bigcap_{a=1}^{n} F_{s_{a}}$,

$$
\Lambda_{v}:=\Lambda_{Q, v}=\left[\lambda\left(F_{s_{1}}\right)^{t}|\ldots| \lambda\left(F_{s_{n}}\right)^{t}\right] .
$$

(6) $G_{E}(v):=\operatorname{ker}\left(\exp \Lambda_{E, v}: T^{n-k} \rightarrow T^{n-k}\right)$.

Notice that the notation $G_{E}(v)$ is used for coker $\Lambda_{E, v}$ in [BSS17, Section 4]. However, the following commutative diagram and snake lemma, see for instance AM69, shows that those two finite groups are isomorphic.

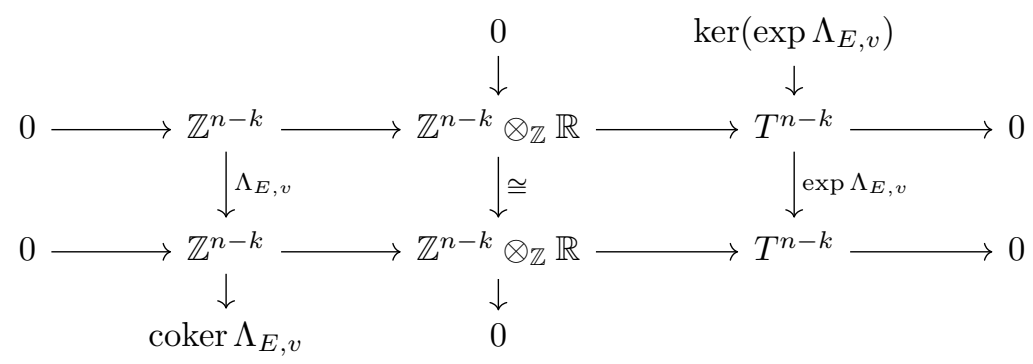

Remark 2.4. One can see from (2.5) that the order $\left|G_{E}(v)\right|$ of the finite group $G_{E}(v)$ is exactly $\left|\operatorname{det} \Lambda_{E, v}\right|$.

Example 2.5. Let $Q$ be a 3 -dimensional prism and $F_{1}, \ldots, F_{5}$ its facets illustrated in Figure 1. We assign $\mathcal{R}$-characteristic vectors by $\lambda\left(F_{1}\right)=(1,0,0), \lambda\left(F_{2}\right)=$ $(0,1,0), \lambda\left(F_{3}\right)=(0,0,1), \lambda\left(F_{4}\right)=(1,2,4)$ and $\lambda\left(F_{5}\right)=(-1,-1,-1)$. Observe that

$$
\begin{aligned}
G_{Q}\left(v_{1}\right) & =\left\{\left(t_{1}, t_{2}, t_{3}\right) \in T^{3} \mid t_{1} t_{3}=t_{3}^{2}=t_{2} t_{3}^{4}=1\right\} \\
& =\langle(1,1,1),(-1,1,-1)\rangle \cong \mathbb{Z} / 2 \mathbb{Z},
\end{aligned}
$$

Similarly we have

$$
\begin{aligned}
G_{Q}\left(v_{2}\right) & =\left\{\left(t_{1}, t_{2}, t_{3}\right) \in T^{3} \mid t_{1} t_{3}=t_{2} t_{3}^{2}=t_{3}^{4}=1\right\} \\
& =\langle(1,1,1),(-i,-1, i),(-1,1,-1),(i,-1,-i)\rangle \cong \mathbb{Z} / 4 \mathbb{Z}, \\
G_{Q}(v) & =\langle(1,1,1)\rangle \cong 1, \text { for } v \in V(Q) \backslash\left\{v_{1}, v_{2}\right\} .
\end{aligned}
$$

Choosing the face $F_{4}$, we consider $\lambda\left(F_{4}\right)=(1,2,4),(0,1,0)$ and $(0,0,1)$ as a basis of $\mathbb{Z}^{3}$. Then, the induced $\mathcal{R}$-characteristic function

$$
\lambda_{F_{4}}:\left\{F_{4} \cap F_{1}, F_{4} \cap F_{2}, F_{4} \cap F_{3}\right\} \rightarrow \mathbb{Z}^{2}
$$


on $F_{4}$ is given by

$$
\lambda_{F_{4}}\left(F_{4} \cap F_{1}\right)=(-1,-2), \lambda_{F_{4}}\left(F_{4} \cap F_{2}\right)=(1,0) \text { and } \lambda_{F_{4}}\left(F_{4} \cap F_{3}\right)=(0,1) .
$$

To be more precise, since $\lambda\left(F_{1}\right)=(1,0,0)=(1,2,4)-2(0,1,0)-4(0,0,1)$, we have $\left(\rho_{F_{4}} \circ \lambda\right)\left(F_{1}\right)=(-2,-4)$ whose primitive vector gives $\lambda_{F_{4}}\left(F_{4} \cap F_{1}\right)$. A similar computation gives other two induced $\mathcal{R}$-characteristic vectors. Moreover, we have

$$
\begin{aligned}
& G_{F_{4}}\left(v_{1}\right)=\left\{\left(t_{1}, t_{2}\right) \in T^{2} \mid t_{1}^{-1}=t_{1}^{-1} t_{2}=1\right\}=\langle(1,1)\rangle \cong 1, \\
& G_{F_{4}}\left(v_{2}\right)=\left\{\left(t_{1}, t_{2}\right) \in T^{2} \mid t_{1}^{-1} t_{2}=t_{2}^{-2}=1\right\}=\langle(1,1),(-1,-1)\rangle \cong \mathbb{Z} / 2 \mathbb{Z} .
\end{aligned}
$$

Finally, $G_{F_{4}}(v)=1$, where $v=F_{2} \cap F_{3} \cap F_{4}$ is considered as a vertex of $F_{4}$.

We finish this section by the following proposition which we shall use in Section 5. One can see this property in Example 2.5.

Proposition 2.6. BSS17, Proposition 4.3] Let $E$ and $E^{\prime}$ be two faces of $Q$ containing a vertex $v$ such that $E$ is a face of $E^{\prime}$. Then, $\left|G_{E}(v)\right|$ divides $\left|G_{E^{\prime}}(v)\right|$.

\section{BUILDING SEQUENCES AND HOMOLOGY OF TORIC ORBIFOLDS}

The goal of this section is to discuss integral homology of toric orbifolds. After Poddar-Sarkar [PS10] developed the notion of q-CW complex, the authors of BNSS introduced a building sequence which enables us to detect $p$-torsion freeness of an orbifold having $\mathbf{q}-\mathrm{CW}$ complex structure for each prime number $p$. In case of toric orbifolds, one can derive a building sequence via a retraction sequence of a simple polytope introduced in BSS17. In this subsection, we review the definition of a building sequence and a retraction sequence and study their relation.

3.1. Building sequence. Let $\bar{D}^{n}$ be a closed $n$-dimensional disc and $G$ a finite group acting linearly on $\bar{D}^{n}$. We call the quotient $\bar{D}^{n} / G$ a $\mathbf{q}$-disc. Now, a q-CW complex is defined inductively in the similar manner as a usual CW complex, but we use q-discs instead of $\bar{D}^{n}$. To be more precise, we start with a discrete set $X_{0}$ of 0-dimensional q-cells. Next, assuming $X_{i-1}$ is defined, we define

$$
X_{i}:=X_{i-1} \cup_{\left\{f_{\alpha}\right\}}\left\{\bar{D}^{i} / G_{\alpha}\right\},
$$

where $f_{\alpha}: \partial\left(\bar{D}^{i} / G_{\alpha}\right) \rightarrow X_{i-1}$ is the attaching map for a q-cell $\bar{D}^{i} / G_{\alpha}$ for finitely many $\alpha$.

A building sequence for a q-CW complex $X$ is a sequence $\left\{Y_{j}\right\}_{j=1}^{\ell}$ of $\mathbf{q}-\mathrm{CW}$ subcomplexes of $X$ such that

$$
Y_{j} \backslash Y_{j-1} \cong D^{k_{j}} / G_{j}
$$

for some $k_{j}$-dimensional open $\mathbf{q}$-disc $D^{k_{j}} / G_{j}$. We denote by $0_{j}$ the image of origin of (3.1) and call it the free special point in $Y_{j}$. When we need to emphasize the free special point, we denote the building sequence by $\left\{\left(Y_{j}, 0_{j}\right)\right\}_{j=1}^{\ell}$, see [BNSS, Section 2] for more details.

Example 3.1. Let $Y_{1}$ be a point and $Y_{2}=Y_{1} \cup_{f_{1}} \bar{D}^{1}$ a circle obtained by the canonical attaching map $f_{1}: S^{0} \rightarrow\{p t\}$. Consider two q-cells $\bar{D}^{2} / \mathbb{Z}_{p}$ and $\bar{D}^{2} / \mathbb{Z}_{q}$, where we regard $\mathbb{Z}_{p}$ as a finite group generated by $p$-th root of unity and it acts on $\bar{D}^{2} \subset \mathbb{C}$ by the multiplication. Similarly we consider $\mathbb{Z}_{q}$-action on $\bar{D}^{2}$. Now we define

$$
Y_{3}:=Y_{2} \cup_{f_{2}} \bar{D}^{2} / \mathbb{Z}_{p} \text { and } Y_{4}:=Y_{3} \cup_{f_{3}} \bar{D}^{2} / \mathbb{Z}_{p}
$$


where $f_{2}: S^{1} / \mathbb{Z}_{p} \rightarrow Y_{1} \cong S^{1}$, respectively $f_{3}: S^{1} / \mathbb{Z}_{q} \rightarrow Y_{1} \subset Y_{2}$, defined by $f_{2}\left(\left[e^{2 \pi i x}\right]\right)=e^{2 \pi i p x}$, respectively $f_{3}\left(\left[e^{2 \pi i y}\right]\right)=e^{2 \pi i q y}$, for $0 \leq x, y \leq 1$. Then, the resulting space $Y_{4}$ is a 2 -dimensional orbifold sphere of football type $\mathbb{C} P_{p, q}^{1}$, see Figure 2,

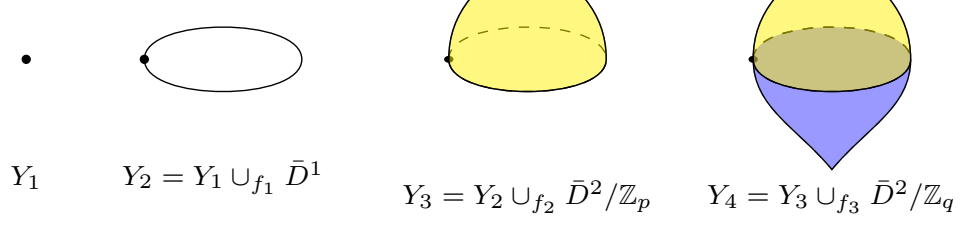

Figure 2. A building sequence of $\mathbb{C} P_{p, q}^{1}$.

Remark 3.2. A q-disc $\bar{D}^{k} / G$ is homeomorphic to the usual disc $\bar{D}^{k}$ if $k=0,1$ or 2. Indeed, the football-type orbifold sphere $\mathbb{C} P_{p, q}^{1}$ is homeomorphic to $S^{2}$ for arbitrary positive integers $p$ and $q$.

3.2. Retraction sequence. Given an $n$-dimensional simple polytope $Q$, as a polytopal complex (see Zie95, Definition 5.1]), we define a sequence of polytopal subcomplexes of $Q$ which will determine a building sequence of a toric orbifold whose orbit space is $Q$. We refer to [BSS17, Section 2] for more details about retraction sequences of a simple polytope.

Let $B$ be a connected polytopal subcomplex of $Q$. A vertex $v$ of $B$ is called a free vertex if $v$ has a neighborhood homeomorphic to $\mathbb{R}_{\geq 0}^{N}$ for some $N \in\{1, \ldots, \operatorname{dim} B\}$ as manifolds with corners. In this case, there exists a unique maximal face $E$ of $B$ containing $v$ as a vertex. We write $F V(B)$ as the set of all free vertices of $B$. For instance, every vertex $v$ of $Q$ is a free vertex and $Q$ itself is the unique (non-proper) face of $Q$ containing $v$. In particular, $F V(Q)=V(Q)$. However, for a polytopal subcomplex $B$ of $Q, F V(B)$ is a subset of $V(B)$ in general.

A retraction sequence $\left\{\left(B_{j}, E_{j}, b_{j}\right)\right\}_{j=1}^{\ell}$ for $Q$ is a sequence of triples consists of a polytopal subcomplex $B_{j}$ of $Q$, a free vertex $b_{j}$ of $B_{j}$ and the unique face $E_{j}$ of $B_{j}$ containing a free vertex $b_{j}$, which is defined inductively such that

$$
B_{j+1}=\bigcup\left\{E \mid E \text { is a face of } B_{j} \text { with } b_{j} \notin E\right\} .
$$

The next term $\left(B_{j+1}, E_{j+1}, b_{j+1}\right)$ is given by the choice of a free vertex $b_{j+1}$ of $B_{j+1}$ and the unique face $E_{j+1}$ determined by the edges of $B_{j+1}$ containing $b_{j+1}$. Finally, the sequence ends up with $\left(B_{\ell}, E_{\ell}, b_{\ell}\right)=\left(b_{\ell}, b_{\ell}, b_{\ell}\right)$ for some vertex $b_{\ell}$ of $Q$.

We note that every simple polytope has at least one retraction sequence. It can be shown by realizing a simple polytope $Q$ as a convex subset in $\mathbb{R}^{n}$ and taking a linear function $f: \mathbb{R}^{n} \rightarrow \mathbb{R}$ such that $f\left(v_{i}\right) \neq f\left(v_{j}\right)$ whenever two vertices $v_{i}$ and $v_{j}$ of $Q$ are distinct. We refer to [BSS17, Proposition 2.3] for the completeness of arguments.

Example 3.3. Let $Q$ be an $\ell$-gon and $v_{1}, \ldots, v_{\ell}$ its vertices with counterclockwise order. Let $F_{i}$ be the 1-dimensional face connecting $v_{i}$ and $v_{i+1}$ for $i=1, \ldots, \ell-1$ and $F_{\ell}$ the 1 -dimensional face with vertices $v_{\ell}$ and $v_{1}$. Then, one canonical choice 

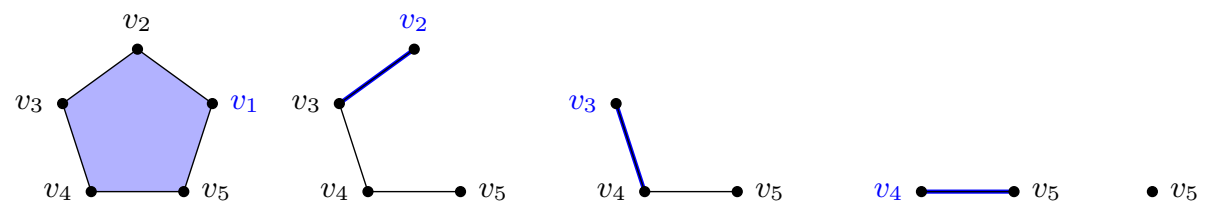

Figure 3. A retraction sequence for 5-gon.

of a retraction sequence $\left\{\left(B_{j}, E_{j}, b_{j}\right)\right\}_{j=1}^{\ell}$ is given by

$$
\left(B_{j}, E_{j}, b_{j}\right)= \begin{cases}\left(Q, Q, v_{1}\right) & \text { if } j=1 ; \\ \left(F_{j} \cup \cdots \cup F_{\ell-1}, F_{j}, v_{j}\right) & \text { if } j=2, \ldots, \ell-1 ; \\ \left(v_{\ell}, v_{\ell}, v_{\ell}\right) & \text { if } j=\ell,\end{cases}
$$

as in Figure 3 for 5-gon.

The following proposition shows a relation between a retraction sequence of a simple polytope $Q$ and a building sequence of the associated toric orbifold $X(Q, \lambda)$.

Proposition 3.4. [BNSS, Proposition 4.4] Let $(Q, \lambda)$ be an $\mathcal{R}$-characteristic pair and $\pi: X(Q, \lambda) \rightarrow Q$ be the orbit map. Then, a retraction sequence $\left\{\left(B_{j}, E_{j}, b_{j}\right)\right\}_{j=1}^{\ell}$ for $Q$ induces a building sequence $\left\{\left(Y_{j}, 0_{j}\right)\right\}_{j=1}^{\ell}$ as follows:

- $Y_{1}=0_{i}=\pi^{-1}\left(b_{\ell}\right)$,

- $Y_{j}=\bigcup_{s=\ell-j+1}^{\ell} \pi^{-1}\left(U_{s}\right)$, where $U_{s}$ is the open subset of $E_{s}$ obtained by deleting faces of $E_{s}$ which does not contain $b_{s}$, for $j=2, \ldots, \ell$.

In particular, $Y_{\ell}=X(Q, \lambda)$ and $0_{j}=\pi^{-1}\left(b_{j}\right)$ for all $j=1, \ldots, \ell$.

Remark 3.5. Though a building sequence induced from a retraction sequence may require the image of an attaching map for a q-cell $\bar{D}^{k} / G$ to be in a higher dimensional skeleton, a $G$-equivariant triangulation [1178, Theorem 3.6] of $\bar{D}^{k} / G$ together with an application of the cellular approximation theorem allows for a deformation into the appropriate dimension.

Next two theorems can be obtained by combining BNSS, Theorem 1.1, 1.2] and Proposition 3.4, which describe a sufficient condition for $(p$-)torsion freeness and vanishing odd degree (co)homology of a toric orbifold.

Theorem 3.6. Let $X:=X(Q, \lambda)$ be a toric orbifold and $p$ a prime number. If there is a retraction $\left\{\left(B_{j}, E_{j}, b_{j}\right)\right\}_{j=1}^{\ell}$ such that $\operatorname{gcd}\left\{p,\left|G_{E_{j}}\left(b_{j}\right)\right|\right\}=1$ for all $j$, then $H_{*}(X ; \mathbb{Z})$ has no $p$-torsion and $H_{\text {odd }}\left(X ; \mathbb{Z}_{p}\right)$ is trivial.

Theorem 3.7. Let $X(Q, \lambda)$ be a toric orbifold. If for each prime $p$, there is a retraction $\left\{\left(B_{j}, E_{j}, b_{j}\right)\right\}_{j=1}^{\ell}$ such that $\operatorname{gcd}\left\{p,\left|G_{E_{j}}\left(b_{j}\right)\right|\right\}=1$ for all $j$, then $H_{*}(X ; \mathbb{Z})$ has no torsion and $H_{\text {odd }}(X ; \mathbb{Z})$ is trivial.

Next two examples are applications of Theorem 3.7 to toric orbifolds over a polygon and a simplex, respectively. Though these results are well known from the literature, for example [Fis92, Jor98, KMZ17] and Kaw73, the same conclusions follow from the results above. 
Example 3.8. Let $Q$ be an $\ell$-gon and $F_{1}, \ldots, F_{\ell}$ facets of $Q$ as in Example 3.3 . Let $\lambda\left(F_{i}\right):=\left(a_{i}, b_{i}\right) \in \mathbb{Z}^{2}$ be characteristic vectors for $i=1, \ldots, \ell$. Hence, we have

$$
\left|G_{Q}\left(v_{1}\right)\right|=\left|\operatorname{det}\left[\begin{array}{ll}
a_{\ell} & a_{1} \\
b_{\ell} & b_{1}
\end{array}\right]\right| \text { and }\left|G_{Q}\left(v_{i}\right)\right|=\left|\operatorname{det}\left[\begin{array}{ll}
a_{i-1} & a_{i} \\
b_{i-1} & b_{i}
\end{array}\right]\right| \text { for } i=2, \ldots, \ell \text {. }
$$

We refer to Remark 2.4. Moreover, since the facets are 1-dimensional, $G_{F_{i}}\left(v_{i}\right)$ is trivial for each facet $F_{i}$. Indeed, one can easily check that the induced $\mathcal{R}$ characteristic function

$$
\lambda_{F_{i}}:\left\{v_{i}, v_{i+1}\right\} \rightarrow \mathbb{Z}
$$

can be always defined by $\lambda_{F_{i}}\left(v_{i}\right)=\lambda_{F_{i}}\left(v_{i+1}\right)= \pm 1$, see Remark 2.3 and Remark 3.2. Now, we assume that

$$
\operatorname{gcd}\left\{\left|G_{Q}\left(v_{1}\right)\right|, \ldots,\left|G_{Q}\left(v_{\ell}\right)\right|\right\}=q .
$$

Then, one can always choose a retraction sequence $\left\{\left(B_{j}, E_{j}, b_{j}\right)\right\}_{j=1}^{\ell}$ of $Q$ such that $\operatorname{gcd}\left\{p,\left|G_{E_{j}}\left(b_{j}\right)\right|\right\}=1$ unless $p$ is a factor of $q$. Hence, we conclude that if $H_{*}(X(Q, \lambda) ; \mathbb{Z})$ has a non-trivial torsion part, then it must have a $p$-torsion for some $p$ dividing $q$. In particular, if $q=1$, then $H_{*}(X(Q, \lambda) ; \mathbb{Z})$ is torsion free.

Example 3.9. Consider an $n$-simplex $\Delta^{n}$ and let $\chi:=\left(\chi_{1}, \ldots, \chi_{n+1}\right) \in \mathbb{N}^{n+1}$. An $\mathcal{R}$-characteristic function

$$
\lambda: \mathcal{F}\left(\Delta^{n}\right):=\left\{F_{1}, \ldots, F_{n+1}\right\} \rightarrow \mathbb{Z}^{n}
$$

satisfying $\sum_{i=1}^{n+1} \chi_{i} \lambda\left(F_{i}\right)=\mathbf{0}$ and $\operatorname{span}_{\mathbb{Z}}\left\{\lambda\left(F_{1}\right), \ldots, \lambda\left(F_{n+1}\right)\right\}=\mathbb{Z}^{n}$. It is well-known that this $\mathcal{R}$-characteristic pair $\left(\Delta^{n}, \lambda\right)$ defines a weighted projective space $\mathbb{C} P_{\chi}^{n}$, see [CLS11, Example 3.1.17] or [Ful93, Section 2.2]. Without loss of generality, we may assume that $\operatorname{gcd}\left\{\chi_{1}, \ldots, \chi_{n+1}\right\}=1$. Indeed, for some $k \in \mathbb{N}$, two vectors $\left(\chi_{1}, \ldots, \chi_{n+1}\right)$ and $\left(k \chi_{1}, \ldots, k \chi_{n+1}\right)$ yield homeomorphic weighted projective spaces, see for example [BFNR13, Theorem 1.1]. Note that $\chi_{i}$ is the order of singularity at $[0, \ldots, 0, \underset{i \text {-th }}{1}, 0, \ldots 0] \in \mathbb{C} P_{\chi}^{n}$, which is same as $\left|G_{\Delta^{n}}\left(v_{i}\right)\right|$ defined from the $\mathcal{R}$-characteristic pair $\left(\Delta^{n}, \lambda\right)$, where $v_{i}=F_{1} \cap \cdots \cap F_{i_{1}} \cap F_{i+1} \cap \cdots F_{n+1}$. Now, following the proof of [BNSS, Proposition 4.5], one can always find retraction sequences $\left\{\left(B_{j}, E_{j}, b_{j}\right)\right\}_{j=1}^{n+1}$ which satisfies the assumption of Theorem 3.7 Hence, we conclude that the (co)homology of any weighted projective space is torsion free and concentrated in even degrees.

\section{J-CONSTRUCTION OF TORIC ORBIFOLDS}

For toric manifolds, the authors of [BBCG15] use a construction introduced in PB80 to construct new toric manifolds from a given one. Indeed, this was done by producing a new characteristic pair from the original one in a canonical way. The process for making a new polytope from the given one is called simplicial wedge construction. Moreover, by a successive procedure, a countably infinite family of new toric manifolds can arise from the original manifold. In BBCG15, where the construction was analyzed in the context of polyhedral products, the process is described efficiently by using a vector $J=\left(j_{1}, \ldots, j_{m}\right) \in \mathbb{N}^{m}$, where $m$ is the number of facets in the original polytope. To be more precise, given a positive integral vector $J=\left(j_{1}, \ldots, j_{m}\right) \in \mathbb{N}^{m}$, one can obtain a new toric manifold $M(J)$ from the original toric manifold $M$. We refer this procedure as the $J$-construction and apply it to toric orbifolds. As an example, we shall investigate the class of spaces that can be produced from a weighted projective spaces. 
4.1. The simplicial wedge construction. Let $K$ be a simplicial complex with vertex set $V(K)=\left\{w_{1}, \ldots, w_{m}\right\}$. We call a subset $\sigma \subset V(K)$ a non-face of $K$ if $\sigma$ is not a simplex in $K$. A non-face $\sigma$ is called minimal if every proper subset of $\sigma$ is a simplex in $K$. Then, the combinatorial type of $K$ is determined by the set of minimal non-faces of $K$.

For arbitrary positive integral vector $J=\left(j_{1}, \ldots, j_{m}\right) \in \mathbb{N}^{m}$, a new simplicial complex $K_{(J)}$ is defined on $V\left(K_{(J)}\right)=\left\{w_{11}, \ldots, w_{1 j_{1}}, \ldots, w_{1 m}, \ldots, w_{m j_{m}}\right\}$ with minimal non-faces of the form $V_{i_{1}} \cup \cdots \cup V_{i_{k}}$, where $V_{i}=\left\{w_{i 1}, \ldots, w_{i j_{i}}\right\}$, whenever $\left\{w_{i_{1}}, \ldots, w_{i_{k}}\right\}$ is a minimal non-face of $K$.

In the special case $J=(1, \ldots, 1, \underset{i-\text { th }}{2}, 1, \ldots, 1)$, we denote $K_{\left(w_{i}\right)}:=K_{(J)}$, and refer to it as the simplicial wedge construction of $K$ on $w_{i} \in V$, see [PB80]. The following representation is a useful combinatorial description of the simplicial wedge construction.

$$
K_{\left(w_{i}\right)}=\left[\left\{w_{i 1}, w_{i 2}\right\} * \operatorname{link}_{K}\left\{w_{i}\right\}\right] \cup\left[\left\{\left\{w_{i 1}\right\},\left\{w_{i 2}\right\}\right\} *\left(K \backslash\left\{w_{i}\right\}\right)\right],
$$

where $*$ denotes the join of two simplicial complexes and we identify $w_{r 1}$ with $w_{r}$ for $r \neq i$.

In this paper, we focus on the case when $K$ is dual to the boundary $\partial Q$ of a simple polytope $Q$, which we denote by $K_{Q}$ and refer to as the nerve complex of $Q$, see for instance [BP15, Section 2.2]. Notice that the vertex set $V\left(K_{Q}\right)=\left\{w_{1}, \ldots, w_{m}\right\}$ bijectively corresponds to the set of facets $\mathcal{F}(Q)=\left\{F_{1}, \ldots, F_{m}\right\}$.

Example 4.1. (1) Let $K=K_{\Delta^{n}}$ be the nerve complex of an $n$-simplex $\Delta^{n}$ and $V(K)=\left\{w_{1}, \ldots, w_{n+1}\right\}$ its vertex set. Then, there exists only one minimal non-face $\sigma=\left\{w_{1}, \ldots, w_{n+1}\right\}$. For an arbitrary $J=\left(j_{1}, \ldots, j_{n+1}\right) \in \mathbb{N}^{n+1}$, $K_{(J)}$ is the simplicial complex on the vertex set

$$
\left\{w_{11}, \ldots, w_{1 j_{1}}, \ldots, w_{n+1,1}, \ldots, w_{n+1, j_{n+1}}\right\}
$$

with the unique minimal non-face $\left\{w_{11}, \ldots, w_{1, j_{1}}, \ldots, w_{n+1,1}, \ldots, w_{n+1, j_{n+1}}\right\}$. Hence, we get $K_{(J)}=K_{\Delta^{d(J)}}$, where $d(J):=\sum_{i=1}^{n+1} j_{i}$. Figure 4 describes the case when $n=2, J=(1,1,2)$ and the decomposition by (4.1).
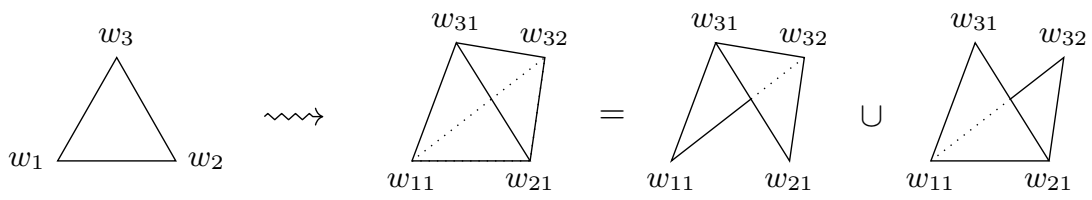

Figure 4. $\left(K_{\Delta^{2}}\right)_{\left(w_{3}\right)}=K_{\Delta^{3}}$.

(2) Consider next the nerve complex

$$
K:=K_{\Delta^{n_{1}} \times \Delta^{n_{2}}}=K_{\Delta^{n_{1}}} * K_{\Delta^{n_{2}}} .
$$

of product of two simplices $\Delta^{n_{1}}$ and $\Delta^{n_{2}}$. Suppose that $\left\{v_{1}, \ldots, v_{n_{1}+1}\right\}$ and $\left\{w_{1}, \ldots, w_{n_{2}+1}\right\}$ are vertex sets of $K_{\Delta^{m}}$ and $K_{\Delta^{n}}$, respectively. Then, $K$ is a simplicial complex on the vertex set $\left\{v_{1}, \ldots, v_{n_{1}+1}, w_{1}, \ldots, w_{n_{2}+1}\right\}$ with two minimal non-faces $\left\{v_{1}, \ldots, v_{n_{1}+1}\right\}$ and $\left\{w_{1}, \ldots, w_{n_{2}+1}\right\}$. Taking $J=(2,1, \ldots, 1) \in \mathbb{N}^{n_{1}+n_{2}+2}$, we obtain

$$
K_{(J)}=K_{\left(v_{1}\right)}=K_{\Delta^{n_{1}+1}} * K_{\Delta^{n_{2}}} .
$$


See Figure 5 for the case when $n_{1}=n_{2}=1$.

$v_{1}$<smiles>C1CCC1</smiles>
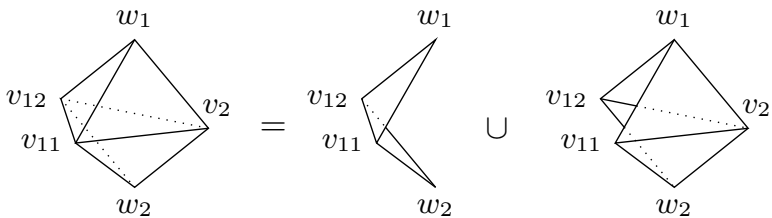

FiguRE 5. $\left(K_{\Delta^{1}} * K_{\Delta^{1}}\right)_{\left(w_{1}\right)}$.

(3) In general, if $K_{1}$ and $K_{2}$ are simplicial complexes on $\left\{v_{1}, \ldots, v_{m_{1}}\right\}$ and $\left\{w_{1}, \ldots, w_{m_{2}}\right\}$, respectively, then

$$
\left(K_{1} * K_{2}\right)_{\left(v_{i}\right)}=K_{1\left(v_{i}\right)} * K_{2} \text { and }\left(K_{1} * K_{2}\right)_{\left(w_{j}\right)}=K_{1} * K_{2\left(w_{j}\right)}
$$

for $i \in\left\{1, \ldots, m_{1}\right\}$ and $j \in\left\{1, \ldots, m_{2}\right\}$. Indeed, one can see these relations by comparing minimal non-faces.

4.2. The polytopal wedge construction. According to [PB80, page 582], if $K$ is a dual to the boundary of a simple polytope $Q$, then $K_{\left(w_{i}\right)}$ is again a simplicial complex which is dual to the boundary of a simple polytope. Notice that, for an arbitrary $J \in \mathbb{N}^{m}, K_{(J)}$ can be obtained by the iterated procedure of simplicial wedge construction. Hence, we can see that $K_{(J)}$ is also dual to the boundary of a simple polytope which we denote by $Q_{(J)}$.

In particular, when $J=J^{\prime}:=(1, \ldots, 1,2,1, \ldots, 1) \in \mathbb{N}^{m}, Q_{\left(J^{\prime}\right)}$ is homeomorphic to

$$
Q_{\left(J^{\prime}\right)}:=(Q \times I) / \sim_{F_{i}}, \text { where }(x, t) \sim_{F_{i}}(y, 0) \text { if } x=y \in F_{i}
$$

as manifolds with corners. Indeed, $(Q \times I) / \sim_{F_{i}}$ has the following facets

$$
\left\{Q^{+}, Q^{-}\right\} \cup\left\{(F \times I) / \sim_{F_{i}} \mid F \in \mathcal{F}(Q) \backslash\left\{F_{i}\right\}\right\}
$$

where $Q^{+}:=Q \times\{1\}$ and $Q^{-}:=Q \times\{0\}$ intersect at $\left(F_{i} \times I\right) / \sim_{F_{i}} \cong F_{i}$ and each of $Q^{+}$and $Q^{-}$intersects all other facets $\left\{(F \times I) / \sim_{F_{i}} \mid F \in \mathcal{F}(Q) \backslash\left\{F_{i}\right\}\right\}$. Notice that this observation is exactly the dual representation of (4.1) given by associating $Q^{-}$, $Q^{+}, F \times I / F_{i}$ for $F \in \mathcal{F}(Q) \backslash\left\{F_{i}\right\}$ with $w_{i 1}, w_{i 2}$ and $w \in V(K) \backslash\left\{w_{i}\right\}$, respectively, where $w$ is the dual of $F$. We call $Q_{\left(J^{\prime}\right)}$ the polytopal wedge construction of $Q$ with respect to $F_{i} \in \mathcal{F}(Q)$. We may also denote $Q_{\left(J^{\prime}\right)}$ by $Q_{\left(F_{i}\right)}$ to emphasize the chosen facet $F_{i}$. See Figure 6 for the example of the polytopal wedge construction of 5-gon.

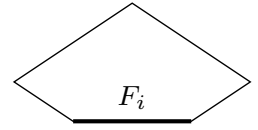

$Q$

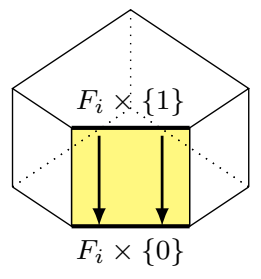

$Q \times I$

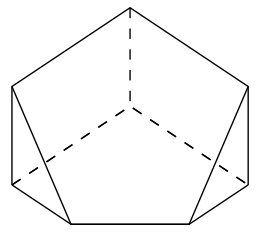

$(Q \times I) / \sim_{F_{i}}$

FiguRE 6. A polytopal wedge construction of 5-gon.

The following example is dual to Example 4.1. 
Example 4.2. (1) $\Delta_{(F)}^{n}=\Delta^{n+1}$, where $F$ is any facet of $\Delta^{n}$.

(2) $\left(\Delta^{n_{1}} \times \Delta^{n_{2}}\right)_{\left(E \times \Delta^{n_{1}}\right)}=\Delta^{n_{1}+1} \times \Delta^{n_{2}}$, where $E$ is a facet of $\Delta^{n_{1}}$, see Figure 5 for the case when $n_{1}=n_{2}=1$.

(3) In general, given two simple polytopes $P$ and $Q$, any facet of $P \times Q$ is of the form $E \times Q$ for some facet $E$ of $P$, or $P \times F$ for some facet $F$ of $Q$. Then, the relation $K_{P \times Q}=K_{P} * K_{Q}$ together with (4.2) leads us to the following:

$$
(P \times Q)_{(E \times Q)}=P_{(E)} \times Q \text { and }(P \times Q)_{(P \times F)}=P \times Q_{(F)} .
$$

4.3. A new $\mathcal{R}$-characteristic function. Let $(Q, \lambda)$ be an $\mathcal{R}$-characteristic pair and $K$ the simplicial complex dual to $\partial Q$ as above. As in previous sections, $m$ and $n$ denote the number of facets of $Q$ and the dimension of $Q$, respectively. Given a vector $J=\left(j_{1}, \ldots, j_{m}\right) \in \mathbb{N}^{m}$, we define a matrix $\Lambda_{(J)}$ of size $\left.(d(J)-m+n)\right) \times d(J)$ as follows, where $d(J):=\sum_{i=1}^{m} j_{i}$;

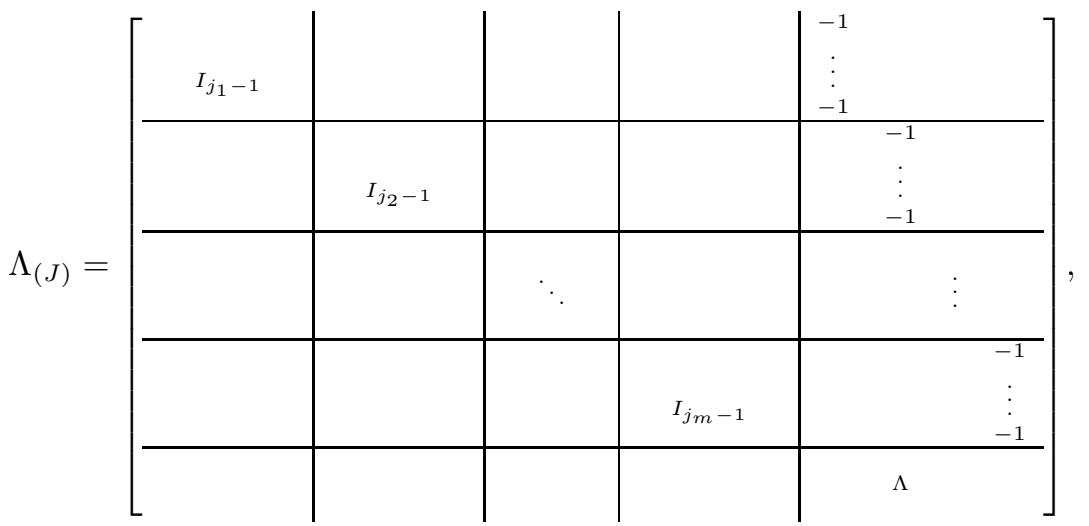

where all the columns of the matrix are indexed respectively by

$$
\left\{w_{12}, \ldots, w_{1 j_{1}}, w_{22}, \ldots, w_{2 j_{2}}, \ldots, w_{m 2}, \ldots, w_{m j_{m}}, w_{11}, \ldots, w_{m 1}\right\},
$$

all of the entries in the empty spaces are zero and $\Lambda$ is the original characteristic matrix associated to $\lambda$. Notice that the indexing in (4.5) bijectively corresponds to the vertex set $V\left(K_{(J)}\right)$.

Let $F_{i k}$ be the facet of $Q_{(J)}$ dual to the vertex $w_{i k}$, where $i \in\{1, \ldots, m\}$ and $k \in\left\{1, \ldots, j_{i}\right\}$. Then, the matrix $\Lambda_{(J)}$ defines a function

$$
\lambda_{(J)}: \mathcal{F}\left(Q_{(J)}\right) \rightarrow \mathbb{Z}^{d(J)-m+n},
$$

by assigning to the facet $F_{i k}$ the transpose of the column vector of $\Lambda_{(J)}$ indexed by $w_{i k}$.

If $\lambda$ satisfies Davis and Januszkiewicz's regularity condition (*), DJ91, p.423], then so does $\lambda_{(J)}$ for all $J \in \mathbb{N}^{m}$, see [BBCG15, Theorem 3.2]. The same proof goes through by replacing condition $(*)$

$$
\operatorname{det}\left[\lambda\left(F_{i_{1}}\right)^{t}|\cdots| \lambda\left(F_{i_{n}}\right)^{t}\right]= \pm 1
$$

with the orbifold condition (2.1)

$$
\operatorname{det}\left[\lambda\left(F_{i_{1}}\right)^{t}|\cdots| \lambda\left(F_{i_{n}}\right)^{t}\right] \neq 0,
$$

to give the next lemma. 
Lemma 4.3. Let $(Q, \lambda)$ be an $\mathcal{R}$-characteristic pair. Then, for arbitrary $J \in \mathbb{N}^{m}$, the function (4.6) satisfies the orbifold condition (2.1).

Hence from $(Q, \lambda)$, one can obtain an infinite family of toric orbifolds

$$
X_{(J)}:=X\left(Q_{(J)}, \lambda_{(J)}\right)
$$

for arbitrary $J \in \mathbb{N}^{m}$.

Example 4.4. Let $Q$ be an $n$-simplex and consider the $\mathcal{R}$-characteristic function from Example [3.9. As in Example 4.1.(1), for an arbitrary vector $J=$ $\left(j_{1}, \ldots, j_{n+1}\right) \in \mathbb{N}^{n+1}$,

$$
\Delta_{(J)}^{n}=\Delta^{d(J)-1} .
$$

Then the function $\lambda_{(J)}$, defined by (4.6), satisfies the equation

$$
\sum_{k=1}^{j_{1}} \chi_{1} \lambda_{(J)}\left(F_{1 k}\right)+\cdots+\sum_{k=1}^{j_{n+1}} \chi_{n} \lambda_{(J)}\left(F_{n+1, k}\right)=\mathbf{0},
$$

and one can show that the new characteristic vectors span the whole lattice $\mathbb{Z}^{d(J)-1}$, since the original $\mathcal{R}$-characteristic vectors in Example 3.9 span $\mathbb{Z}^{n}$. Hence, we conclude that $\left(\mathbb{C} P_{\chi}^{n}\right)_{(J)}$ is the weighted projective space $\mathbb{C} P_{\chi(J)}^{d(J)-1}$, where

$$
\chi_{(J)}=(\underbrace{\chi_{1}, \ldots, \chi_{1}}_{j_{1}}, \ldots, \underbrace{\chi_{k}, \ldots, \chi_{k}}_{j_{k}}, \ldots, \underbrace{\chi_{n+1}, \ldots, \chi_{n+1}}_{j_{n+1}}) \in \mathbb{N}^{d(J)} .
$$

In the special case $J^{\prime}:=(1, \ldots, 1,2,1, \ldots, 1)$, the matrix of (4.4) takes a particularly simple form,

$$
\Lambda_{\left(J^{\prime}\right)}=\left[\begin{array}{cccccc}
1 & 0 & \cdots & -1 & \cdots & 0 \\
0 & & & & & \\
\vdots & \lambda\left(F_{1}\right) & \cdots & \lambda\left(F_{i}\right) & \cdots & \lambda\left(F_{m}\right) \\
0 & & & & &
\end{array}\right] .
$$

Hence, the characteristic function $\lambda_{\left(J^{\prime}\right)}: \mathcal{F}\left(Q_{\left(J^{\prime}\right)}\right) \rightarrow \mathbb{Z}^{n+1}$ is defined by

$$
\begin{aligned}
& \lambda_{\left(J^{\prime}\right)}\left(Q^{+}\right)=(1,0, \ldots, 0)^{t}, \\
& \lambda_{\left(J^{\prime}\right)}\left(Q^{-}\right)=\left(-1, \lambda\left(F_{i}\right)\right)^{t} \text { and } \\
& \lambda_{\left(J^{\prime}\right)}\left(F_{s 1}\right)=\left(-1, \lambda\left(F_{s}\right)\right)^{t}, \text { for } s \in\{1, \ldots, i-1, i+1, \ldots, m\} .
\end{aligned}
$$

Remark 4.5. The two induced $\mathcal{R}$-characteristic functions $\lambda_{\left(J^{\prime}\right)_{Q^{+}}}$and $\lambda_{\left(J^{\prime}\right)_{Q^{-}}}$ coincide with $\lambda$. This implies that $X_{(2,1, \ldots, 1)}$ has two copies of original orbifold $X$ as suborbifolds defined in Section 2. Ewald [Ewa86] called $X_{(2,1, \ldots, 1)}$ the canonical extension of $X$, when $X$ is a toric variety. We refer to [BBCG, BBCG15, CP16, CP17 and Ewa86] for more topological and geometrical observations about the wedge operation on toric manifolds.

The following proposition confirms that $X_{(J)}$ for arbitrary $J=\left(j_{1}, \ldots, j_{m}\right) \in$ $\mathbb{N}^{m}$ can be constructed from iterated wedge operations as mentioned in BBCG15, Remark 3.1]. Here, we give an explicit proof.

Proposition 4.6. Let $X$ be the toric orbifold associated to an $\mathcal{R}$-characteristic pair $(Q, \lambda)$. Then, two toric orbifolds $X_{(3,1, \ldots, 1)}$ and $Y_{(2,1, \ldots, 1)}$ where $Y=X_{(2,1, \ldots, 1)}$ are homeomorphic. 
Proof. Let $\left\{w_{1}, \ldots, w_{m}\right\}$ be the vertices of $K:=K_{Q}$. Then, one can see from the definition of $K_{(J)}$ or (4.1) that both $K_{(3,1, \ldots, 1)}$ and $\left(K_{(2,1, \ldots, 1)}\right)_{(2,1, \ldots, 1)}$ have the same number of vertices and have the same minimal non faces. Therefore, the simple polytopes $Q_{(3,1, \ldots, 1)}$ and $\left(Q_{(2,1 \ldots, 1)}\right)_{(2,1, \ldots, 1)}$ determined by $K_{(3,1, \ldots, 1)}$ and $\left(K_{(2,1, \ldots, 1)}\right)_{(2,1, \ldots, 1)}$, respectively, are homeomorphic as manifolds with corners.

The two characteristic matrices $\Lambda_{(3,1, \ldots, 1)}$ and $\left(\Lambda_{(2,1, \ldots, 1)}\right)_{(2,1, \ldots, 1)}$ differ by an element

$$
\left(\begin{array}{ccccc}
1 & -1 & 0 & \cdots & 0 \\
0 & 1 & 0 & \cdots & 0 \\
\vdots & & \ddots & & \vdots \\
\vdots & & & 1 & 0 \\
0 & \cdots & \cdots & 0 & 1
\end{array}\right) \in S L_{n+2}(\mathbb{Z})
$$

which induces an automorphism

$$
\phi: T^{n+2} \rightarrow T^{n+2}
$$

given by $\phi\left(t_{1}, \ldots, t_{n+2}\right)=\left(t_{1} t_{2}^{-1}, t_{2}, \ldots, t_{n+2}\right)$. Finally, the map

$$
\phi \times i d: T^{n+2} \times Q_{(3,1, \ldots, 1)} \rightarrow T^{n+2} \times\left(Q_{(2,1 \ldots, 1)}\right)_{(2,1, \ldots, 1)}
$$

induces a homeomorphism from $X_{(3,1, \ldots, 1)}$ to $\left(X_{(2,1, \ldots, 1)}\right)_{(2,1, \ldots, 1)}$.

\section{Homology of $X_{(J)}$}

In this section, we shall see that the homology of $X_{(J)}$ depends on $J$ and the homology of $X$. First we compare retraction sequences for the two polytopes $Q$ and $Q_{(J)}$. It suffices to consider the case when $J^{\prime}=(1, \ldots, 1,2,1, \ldots, 1)$, because $Q_{(J)}$ can be constructed by the iterations of the polytopal wedge construction, as in Subsection 4.2. We assume that the entry 2 appears in $i$-th coordinate of $J^{\prime}$, hence it corresponds to the $i$-th facet $F_{i}$ of $Q$.

Let $V(Q)=\left\{v_{1}, \ldots, v_{\ell}\right\}$ be the vertices of $Q$ and $V\left(F_{i}\right):=\left\{v_{i_{1}}, \ldots, v_{i_{k}}\right\}$ the vertices of $F_{i}$. Now, the vertices of $Q_{\left(J^{\prime}\right)}$ are identified as

$$
V\left(Q_{\left(J^{\prime}\right)}\right)=\left\{v^{+}, v^{-} \mid v \in V(Q) \backslash V\left(F_{i}\right)\right\} \cup\left\{v_{i_{1}}^{-}, \ldots, v_{i_{k}}^{-}\right\},
$$

where we write $v^{+}:=v \times\{1\}$ and $v^{-}:=v \times\{0\}$ for notational convenience.

Now, we introduce the following two lemmas about the finite group $G_{Q_{\left(J^{\prime}\right)}}(u)$, as defined in Section 2, associated to each vertex $u$ of $Q_{\left(J^{\prime}\right)}$.

Lemma 5.1. For each vertex $v_{i_{r}} \in V\left(F_{i}\right), r \in\{1, \ldots, k\}$, the finite group $G_{Q_{\left(J^{\prime}\right)}}\left(v_{i_{r}}^{-}\right)$ is isomorphic to $G_{Q}\left(v_{i_{r}}\right)$.

Proof. Given a vertex $v_{i_{r}} \in V\left(F_{i}\right)$, assume that $v_{i_{r}}=F_{i} \cap F_{s_{1}} \cap \cdots \cap F_{s_{n-1}}$ for some $\left\{s_{1}, \ldots, s_{n-1}\right\} \subset\{1, \ldots, m\}$. Then, we have

$$
v_{i_{r}}^{-}=Q^{+} \cap Q^{-} \cap \bigcap_{a=1}^{n-1} F_{s_{a}\left(F_{i} \cap F_{s_{a}}\right)},
$$

where $F_{s_{a}\left(F_{i} \cap F_{s_{a}}\right)}$ is the polytopal wedge construction, as in Subsection 4.2, by regarding the facet $F_{s_{a}}$ as a simple polytope, and $F_{i} \cap F_{s_{a}}$ as a facet of $F_{s_{a}}$ for $a=1, \ldots, n-1$. 
Restricting to the facets which meet at $v_{i_{r}}^{-}$, we get from (4.7)

$$
\begin{aligned}
& \Lambda_{\left(J^{\prime}\right)_{v_{i_{r}}^{-}}}=\left[\lambda_{\left(J^{\prime}\right)}\left(Q^{+}\right)^{t}\left|\lambda_{\left(J^{\prime}\right)}\left(Q^{-}\right)^{t}\right| \lambda_{\left(J^{\prime}\right)}\left(F_{s_{1}\left(F_{i} \cap F_{s_{1}}\right)}\right)^{t}|\cdots| \lambda_{\left(J^{\prime}\right)}\left(F_{s_{n-1}\left(F_{i} \cap F_{s_{n-1}}\right)}\right)^{t}\right] \\
& =\left[\begin{array}{ccccc}
1 & -1 & 0 & \cdots & 0 \\
0 & & & & \\
\vdots & \lambda\left(F_{i}\right)^{t} & \lambda\left(F_{s_{1}}\right)^{t} & \cdots & \lambda\left(F_{s_{n-1}}\right)^{t}
\end{array}\right],
\end{aligned}
$$

which induces an endomorphism $\exp \left(\Lambda_{\left(J^{\prime}\right)}{v_{i_{r}}^{-}}\right): T^{n+1} \rightarrow T^{n+1}$. According to the definition in Page 5 of Section 2, we have

$$
\begin{aligned}
G_{Q_{\left(J^{\prime}\right)}}\left(v_{i_{r}}^{-}\right) & =\operatorname{ker}\left(\exp \left(\Lambda_{\left(J^{\prime}\right)}{v_{i_{r}}^{-}}\right): T^{n+1} \rightarrow T^{n+1}\right) \\
& =\left\{\left(t_{1}, \ldots, t_{n+1}\right) \in T^{n+1} \mid t_{1}=t_{2},\left(t_{2}, \ldots, t_{n+1}\right) \in G_{Q}\left(v_{i_{r}}\right)\right\} \\
& \cong G_{Q}\left(v_{i_{r}}\right) .
\end{aligned}
$$

Next, we consider vertices away from $F_{i}$.

Lemma 5.2. Let $v$ be a vertex in $V(Q) \backslash V\left(F_{i}\right)$. Then, the finite groups $G_{Q_{\left(J^{\prime}\right)}}\left(v^{+}\right)$ and $G_{Q_{\left(J^{\prime}\right)}}\left(v^{-}\right)$are isomorphic to $G_{Q}(v)$.

Proof. Suppose $v=F_{s_{1}} \cap \cdots \cap F_{s_{n}}$ with $i \notin\left\{s_{1}, \ldots, s_{n}\right\}$. Then, we have

$$
v^{-}=Q^{-} \cap\left(F_{s_{1}} \times I\right) \cap \cdots \cap\left(F_{s_{n}} \times I\right) \text {. }
$$

The $\mathcal{R}$-characteristic function $\lambda_{\left(J^{\prime}\right)}$ yields the square matrix

$$
\begin{aligned}
& \Lambda_{\left(J^{\prime}\right)_{v^{-}}}=\left[\lambda_{\left(J^{\prime}\right)}\left(Q^{-}\right)^{t}\left|\lambda_{\left(J^{\prime}\right)}\left(F_{s_{1}} \times I\right)^{t}\right| \cdots \mid \lambda_{\left(J^{\prime}\right)}\left(F_{s_{n}} \times I\right)^{t}\right] \\
& =\left[\begin{array}{c|c|c|c}
-1 & 0 & \cdots & 0 \\
\lambda\left(F_{i}\right)^{t} & \lambda\left(F_{s_{1}}\right)^{t} & \cdots & \lambda\left(F_{s_{n}}\right)^{t}
\end{array}\right] \\
& =\left[\begin{array}{c|ccc}
-1 & 0 & \cdots & 0 \\
\hline \lambda\left(F_{i}\right)^{t} & & \Lambda_{v} & \\
& & &
\end{array}\right] .
\end{aligned}
$$

Hence the kernel of the endomorphism $\exp \left(\Lambda_{\left(J^{\prime}\right)_{v^{-}}}\right): T^{n+1} \rightarrow T^{n+1}$ of tori induced by $\Lambda_{\left(J^{\prime}\right)_{v^{-}}}$is

$$
\left\{\left(t_{1}, \ldots, t_{n+1}\right) \in T^{n+1} \mid t_{1}=1,\left(t_{2}, \ldots, t_{n+1}\right) \in G_{Q}(v)\right\}
$$

which is isomorphic to $G_{Q}(v)$. Similarly one can show that $G_{Q_{\left(J^{\prime}\right)}}\left(v^{+}\right)$is also isomorphic to $G_{Q}(v)$.

The observations above allow us now to adapt the hypothesis of Theorem 3.6 to the $\mathcal{R}$-characteristic pair $\left(Q_{(J)}, \lambda_{(J)}\right)$.

Lemma 5.3. Given an $\mathcal{R}$-characteristic pair $(Q, \lambda)$ and a prime number $p$, suppose that there exist a retraction sequence $\left\{\left(B_{r}, E_{r}, b_{r}\right)\right\}_{r=1}^{\ell}$ of $Q$ satisfying the condition $\operatorname{gcd}\left\{p,\left|G_{E_{r}}\left(b_{r}\right)\right|\right\}=1$ for $r=1, \ldots, \ell$. Then for an arbitrary $J=\left(j_{1}, \ldots, j_{m}\right) \in$ $\mathbb{N}^{m}$, there exists a retraction sequence $\left\{\left(B_{s}^{\prime}, E_{s}^{\prime}, b_{s}^{\prime}\right)\right\}_{s=1}^{\ell^{\prime}}$ for $Q_{(J)}$ which satisfies $\operatorname{gcd}\left\{p,\left|G_{E_{s}^{\prime}}\left(b_{s}^{\prime}\right)\right|\right\}=1$ for $s=1, \ldots, \ell^{\prime}$, where $\ell^{\prime}:=\left|V\left(Q_{(J)}\right)\right|$. 
Proof. It is enough to consider the case when $J=J^{\prime}=(1, \ldots, 1,2,1, \ldots, 1)$ from the discussion in Section 4 and the opening remark of Section 5. We assume the entry 2 appears in $i$-th coordinate of $J$ and let $b_{\beta_{1}} \ldots, b_{\beta_{k}}$ be the vertices of the facet $F_{i}$ of $Q$, where $\beta_{1}<\cdots<\beta_{k}$.

Given a prime number $p$ and a retraction sequence $\left\{\left(B_{r}, E_{r}, b_{r}\right)\right\}_{r=1}^{\ell}$ for $Q$ such that $\operatorname{gcd}\left\{p,\left|G_{E_{r}}\left(b_{r}\right)\right|\right\}=1$ for $r=1, \ldots, \ell$, we now construct a retraction sequence $\left\{\left(B_{s}^{\prime}, E_{s}^{\prime}, b_{s}^{\prime}\right)\right\}_{s=1}^{2 \ell-k}$ for $Q_{\left(J^{\prime}\right)}$ satisfying the hypothesis. To accomplish this, consider the following sequence of vertices of $Q_{\left(J^{\prime}\right)}$ :

$$
\begin{gathered}
b_{1}^{+} \rightarrow b_{1}^{-} \rightarrow \cdots \rightarrow b_{\beta_{1}-1}^{+} \rightarrow b_{\beta_{1}-1}^{-} \rightarrow b_{\beta_{1}}^{-} \rightarrow b_{\beta_{1}+1}^{+} \rightarrow b_{\beta_{1}+1}^{-} \rightarrow \cdots \\
\cdots \rightarrow b_{\beta_{k}-1}^{+} \rightarrow b_{\beta_{k}-1}^{-} \rightarrow b_{\beta_{k}}^{-} \rightarrow b_{\beta_{k}+1}^{+} \rightarrow b_{\beta_{k}+1}^{-} \rightarrow \cdots \rightarrow b_{\ell}^{+} \rightarrow b_{\ell}^{-} .
\end{gathered}
$$

The sequence (5.4) begins with $b_{1}^{-}$if $b_{1}$ is a vertex of $F_{i}$, i.e., $\beta_{1}=1$. Now, we construct a retraction sequence satisfying the hypothesis using the sequence (5.4) above.

(Case 1) We first assume that $b_{1}$ is a vertex of $F_{i}$. Then, we take $b_{1}^{\prime}=b_{1}^{-}$as in (5.4) and set $\left(B_{1}^{\prime}, E_{1}^{\prime}, b_{1}^{\prime}\right)=\left(Q_{\left(J^{\prime}\right)}, Q_{\left(J^{\prime}\right)}, b_{1}^{-}\right)$. Next, the choice of $b_{1}^{-}$as a free vertex of $B_{1}^{\prime}$ gives

$$
B_{2}^{\prime}=\left(\bigcup_{E \cap F_{i}=\emptyset} E \times I\right) \cup\left(\bigcup_{\substack{E \cap F_{i} \neq \emptyset \\ b_{1} \notin E}} E_{\left(E \cap F_{i}\right)}\right),
$$

where $E$ is a face of $Q$, and $E_{\left(E \cap F_{i}\right)}$ is the polytopal wedge of $E$ by considering $E$ as a simple polytope and $E \cap F_{i}$ as a facet of $E$. In general, if a face $E$ of a simple polytope $Q$ intersects a facet $F$ of $Q$, then $E$ is a face of $F_{i}$ or $E \cap F_{i}$ is a facet of $E$. Observe that the face structure of $B_{2}^{\prime}$ is naturally inherited from the face structure of $Q_{\left(J^{\prime}\right)}$. In particular, neither $Q^{+}$nor $Q^{-}$is a face of $B_{2}^{\prime}$.

Next, we consider the following two possibilities: (i) $b_{2} \in V\left(F_{i}\right) \backslash\left\{b_{1}\right\}$, i.e., $\beta_{2}=2$, and (ii) $b_{2} \notin V\left(F_{i}\right)$. If $b_{2} \in V\left(F_{i}\right) \backslash\left\{b_{1}\right\}$, we set $b_{2}^{\prime}=b_{2}^{-}$and $E_{2}^{\prime}=E_{2\left(E_{2} \cap F_{i}\right)}$. If $b_{2} \notin V\left(F_{i}\right)$, we set $b_{2}^{\prime}=b_{2}^{+}$and $E_{2}^{\prime}=E_{2} \times I$. Then, $b_{2}^{\prime}$ has a neighborhood homeomorphic to $\mathbb{R}_{\geq 0}^{\operatorname{dim} E_{2}+1}$ in $E_{2}^{\prime}$, because $b_{2}$ has the appropriate neighborhood in $E_{2}$. Hence, we can define the second term $\left(B_{2}^{\prime}, E_{2}^{\prime}, b_{2}^{\prime}\right)$. The first two retraction sequences in Figure 7 illustrate this case when $Q$ is a pentagon.

(Case 2) Here we assume that $b_{1} \notin V\left(F_{i}\right)$. Then, we take $b_{1}^{\prime}=b_{1}^{+}$by (5.4), which gives

$$
B_{2}^{\prime}=Q^{-} \cup\left(\bigcup_{E \cap F_{i}=\emptyset} E \times I\right) \cup\left(\bigcup_{\substack{E \cap F_{i} \neq \emptyset \\ b_{1} \notin E}} E_{\left(E \cap F_{i}\right)}\right),
$$

where $E$ is a face of $Q$. Next, we take $b_{2}^{\prime}=b_{1}^{-}$and $E_{2}^{\prime}=Q^{-}$which is the unique maximal face of $Q_{\left(J^{\prime}\right)}$ containing $b_{2}^{\prime}$. Now, $B_{3}^{\prime}$ is naturally defined by deleting faces of $Q^{-}$which contains $b_{2}^{\prime}$ from $B_{2}^{\prime}$. Observe that neither $Q^{+}$nor $Q^{-}$is a face of $B_{3}^{\prime}$, because a vertex in each of $Q^{+}$and $Q^{-}$has been removed. See the third retraction sequence in Figure 7 for an example.

In both (Case1) and (Case2), one can see that

$$
\operatorname{gcd}\left\{p,\left|G_{E_{1}^{\prime}}\left(b_{1}^{\prime}\right)\right|\right\}=\operatorname{gcd}\left\{p,\left|G_{E_{2}^{\prime}}\left(b_{2}^{\prime}\right)\right|\right\}=1
$$


Case 1-(i):

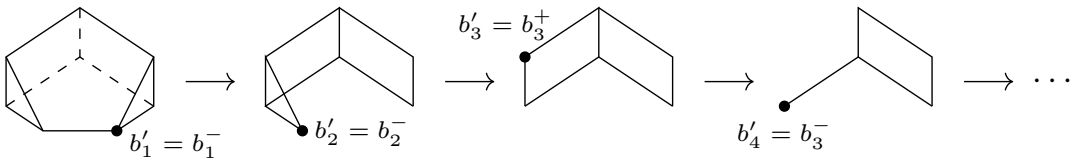

Case 1-(ii):
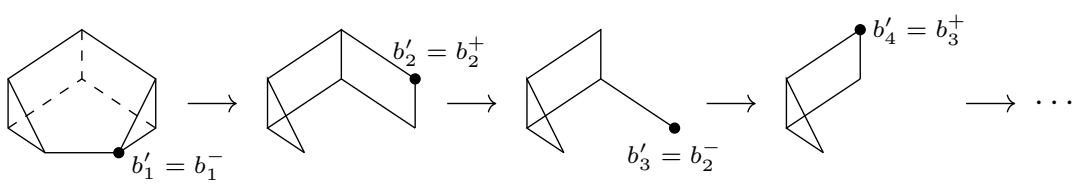

Case 2:
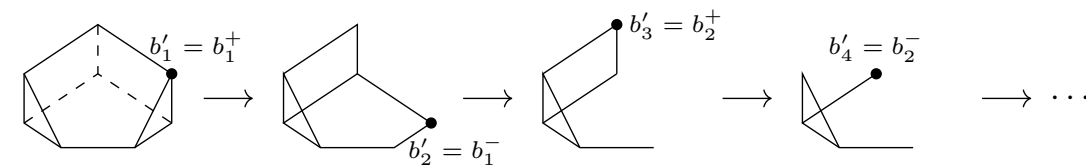

FiguRE 7. Three retraction sequences of the wedge of 5-gon.

by Proposition 2.6, Lemma 5.1 and Lemma 5.2.

Finally, the remaining terms of the desired retraction sequence $\left\{\left(B_{s}^{\prime}, E_{s}^{\prime}, b_{s}^{\prime}\right)\right\}_{s=1}^{2 \ell-k}$ can be obtained by setting the vertices in the sequence (5.4) as the desired sequence of free vertices $\left\{b_{s}^{\prime}\right\}_{s=1}^{2 \ell-k}$. Indeed, it is enough to verify the following claims:

(a) Assume that $b_{r} \notin V\left(F_{i}\right), b_{s}^{\prime}=b_{r}^{+}$and $b_{s+1}^{\prime}=b_{r}^{-}$. Then, $b_{s}^{\prime}$ and $b_{s+1}^{\prime}$ are free vertices in $B_{s}^{\prime}$ and $B_{s+1}^{\prime}$, respectively. In particular, $E_{s}^{\prime}$ and $E_{s+1}^{\prime}$ are determined by $E_{r} \times I$ and $E_{r} \times\{0\}$, respectively.

(b) Assume that $b_{r} \in V\left(F_{i}\right)$ and $b_{s}^{\prime}=b_{r}^{-}$. Then, $b_{s}^{\prime}$ is a free vertex in $B_{s}^{\prime}$ with a unique maximal face $E_{s}^{\prime}=E_{r\left(E_{r} \cap F_{i}\right)}$.

(c) $\operatorname{gcd}\left\{p,\left|G_{E_{s}^{\prime}}\left(b_{s}^{\prime}\right)\right|\right\}=1$ for each $s=1, \ldots, \ell^{\prime}$.

The claims follow by repeating the arguments in (Case 1) and (Case 2) above.

Now, Theorem 3.6, 3.7 and Lemma 5.3 concludes the following two theorems.

Theorem 5.4. Let $X:=X(Q, \lambda)$ be a toric orbifold satisfying the assumption of Theorem 3.6. Then the cohomology $H^{*}\left(X_{(J)} ; \mathbb{Z}\right)$ has no $p$-torsion and $H_{\text {odd }}\left(X_{(J)} ; \mathbb{Z}_{p}\right)$ is trivial for arbitrary $J=\left(j_{1}, \ldots, j_{m}\right) \in \mathbb{N}^{m}$.

Theorem 5.5. Let $X:=X(Q, \lambda)$ be a toric orbifold satisfying the assumption of Theorem 3.7. Then, $H^{*}\left(X_{(J)} ; \mathbb{Z}\right)$ is torsion free and $H_{\text {odd }}\left(X_{(J)} ; \mathbb{Z}\right)$ vanishes for arbitrary $J=\left(j_{1}, \ldots, j_{m}\right) \in \mathbb{N}^{m}$.

\section{Applichtion to the Cohomology Ring}

Motivated by the results of [BFR09], a notion of weighted Stanley-Reisner ring was introduced and used in BSS17 to explicitly compute the singular cohomology ring with integer coefficients of spaces identified as integrally equivariantly formal projective toric orbifolds. In this section, we briefly introduce their results and study the integral cohomology ring of $X_{(J)}$.

6.1. Cohomology ring of $X$. In this subsection, we briefly summarize the theory of weighted Stanley-Reisner ring of a simple lattice polytop 3 $Q$ which defines a

\footnotetext{
${ }^{3} \mathrm{~A}$ simple polytope in $\mathbb{R}^{n}$ whose vertices belong to the lattice $\mathbb{Z}^{n} \subset \mathbb{R}^{n}$.
} 
projective normal toric variety, see [BSS17, Section 5]. The original definition of weighted Stanley-Reisner ring is based on a polytopal fan, but we translate the notation of [BSS17] to a simple lattice polytope which defines a polytopal fan.

Let $\mathcal{F}(Q)=\left\{F_{1}, \ldots, F_{m}\right\}$ be the set of facets of $Q$. Since $Q$ is a lattice polytope, we may choose a primitive inward normal vector $\lambda_{i}$ of each facet $F_{i}$. Moreover, if $F_{i_{1}} \cap \cdots \cap F_{i_{k}}=\emptyset$, then $\lambda_{i_{1}}, \ldots, \lambda_{i_{k}}$ is linearly independent, because $Q$ is simple. Hence, the set of primitive inward normal vectors forms an $\mathcal{R}$-characteristic pair, say $(Q, \lambda)$.

Next, for each vertex $v=F_{s_{1}} \cap \cdots \cap F_{s_{n}} \in V(Q)$, we recall from Section 2, (item (5) in page 5), the $(n \times n)$-matrix associated to $v$, that is

$$
\Lambda_{v}:=\left[\lambda\left(F_{s_{1}}\right)^{t}|\cdots| \lambda\left(F_{s_{n}}\right)^{t}\right] .
$$

For each vertex $v=F_{s_{1}} \cap \cdots \cap F_{s_{n}}$, we define a vector

$$
z^{v}:=\left(z_{1}^{v}, \ldots, z_{m}^{v}\right) \in \bigoplus_{m} \mathbb{Q}\left[u_{1}, \ldots, u_{n}\right]
$$

by the following rule:

(i) $z_{j}^{v}=0$ if $j \notin\left\{s_{1}, \ldots, s_{n}\right\}$,

(ii) $\left[\begin{array}{c}z_{s_{1}}^{v} \\ \vdots \\ z_{s_{n}}^{v}\end{array}\right]=\Lambda_{v}^{-1} \cdot\left[\begin{array}{c}u_{1} \\ \vdots \\ u_{n}\end{array}\right]$

where the operation on the right hand side is the usual matrix multiplication. Next, we define a subset of $\mathbb{Z}\left[x_{1}, \ldots, x_{m}\right]$ as follows:

$$
\operatorname{Int}[Q, \lambda]:=\left\{f\left(x_{1}, \ldots, x_{m}\right) \mid f\left(z^{v}\right) \in \mathbb{Z}\left[u_{1}, \ldots, u_{n}\right], \text { for all } v \in V(Q)\right\} .
$$

Remark 6.1. The variables $u_{1}, \ldots, u_{n}$ stand for the basis of $H^{2}\left(B T^{n} ; \mathbb{Z}\right)$, where $T^{n}$ is the $n$-dimensional torus acting on $X(Q, \lambda)$. Indeed, one may regard polynomial rings $\mathbb{Q}\left[u_{1}, \ldots, u_{n}\right]$ and $\mathbb{Z}\left[u_{1}, \ldots, u_{n}\right]$ as $H^{*}\left(B T^{n} ; \mathbb{Q}\right)$ and $H^{*}\left(B T^{n} ; \mathbb{Z}\right)$, respectively. We refer to [BSS17, Section 5.2].

The next proposition highlights critical properties of $\operatorname{Int}[Q, \lambda]$.

Proposition 6.2. (1) The subset $\operatorname{Int}[Q, \lambda]$ is a subring of $\mathbb{Z}\left[x_{1}, \ldots, x_{m}\right]$.

(2) The Stanley-Reisner ideal $\mathcal{I}_{Q}:=\left\langle\prod_{j=1}^{k} x_{i_{j}} \mid F_{i_{1}} \cap \cdots \cap F_{i_{k}}=\emptyset\right\rangle$ of the ring $\mathbb{Z}\left[x_{1}, \ldots, x_{m}\right]$ for $Q$ is again an ideal of $\operatorname{Int}[Q, \lambda]$.

Proof. The first statement is almost obvious. Next, if a monomial $f\left(x_{1}, \ldots x_{m}\right)=$ $\prod_{j=1}^{k} x_{i_{j}}$ is an element of $\mathcal{I}_{Q}$, it follows from item (i) above that $f\left(z^{v}\right)=0$ for all $v \in V(Q)$. Hence, $\mathcal{I}_{Q}$ is not only a subset, but also an ideal of $\operatorname{Int}[Q, \lambda]$.

Definition 6.3. BSS17, Section 5] The weighted Stanley-Reisner ring $w \mathcal{S R}[Q, \lambda]$ of an $\mathcal{R}$-characteristic pair $(Q, \lambda)$, associated to a simple lattice polytope, is the subring of the Stanley-Reisner ring $\mathcal{S R}[Q]$ of $Q$ defined by the quotient:

$$
w \mathcal{S R}[Q, \lambda]:=\operatorname{Int}[Q, \lambda] / \mathcal{I}_{Q}
$$

We remark that if a simple lattice polytope is Delzant, i.e., normal vectors associated to facets intersecting a vertex form a $\mathbb{Z}$-basis, then $\Lambda_{v}^{-1}$ has integer entries. Hence, $f\left(z^{v}\right)$ is polynomial with integer coefficients for all $v \in V(Q)$, which says that $w \mathcal{S R}[Q, \lambda]$ is the usual Stanley-Reisner ring of a simple lattice polytope. Hence, $w \mathcal{S} \mathcal{R}[Q, \lambda]$ contains geometric data about $X(Q, \lambda)$, including singularities, in addition to the combinatorial information about $Q$. 
Theorem 6.4. BSS17, Theorem 5.3] Let $X(Q, \lambda)$ be a projective toric variety over a simple lattice polytope $Q$ with $H^{\text {odd }}(X(Q, \lambda) ; \mathbb{Z})=0$. Then, there is an isomorphism

$$
H^{*}(X(Q, \lambda) ; \mathbb{Z}) \cong w \mathcal{S} \mathcal{R}[Q, \lambda] / \mathcal{J}
$$

where $\mathcal{J}$ is the ideal generated by the linear elements $\sum_{i=1}^{m}\left\langle\lambda_{i}, \mathbf{e}_{j}\right\rangle x_{i}$ for $j=$ $1, \ldots, n$, where $\mathbf{e}_{j}$ denotes the $j$-th standard unit vector in $\mathbb{Z}^{n}$.

6.2. The cohomology ring of $X_{(J)}$. In this final section, we study the relationship between the cohomology ring of $X$ and that of $X_{(J)}:=X\left(Q_{(J)}, \lambda_{(J)}\right)$. The next theorem follows directly from Theorem 3.7 and Theorem 6.4 .

Theorem 6.5. Let $(Q, \lambda)$ be an $\mathcal{R}$-characteristic pair satisfying the hypothesis of Theorem 3.7. Then,

$$
H^{*}\left(X_{(J)} ; \mathbb{Z}\right) \cong w \mathcal{S} \mathcal{R}\left[Q_{(J)}, \lambda_{(J)}\right] / \mathcal{J}_{(J)},
$$

where $\mathcal{J}_{(J)}$ is the ideal generated by

$$
\left\{\sum_{i=1}^{m}\left\langle\lambda_{i}, e_{j}\right\rangle x_{i 1} \mid j=1, \ldots, n\right\} \cup\left\{x_{i t}=x_{i 1} \mid t=2, \ldots, j_{i}\right\}
$$

If $X$ is a smooth toric manifold, then the cohomology ring $H^{*}\left(X_{(J)} ; \mathbb{Z}\right)$ can be dramatically simplified, because $w \mathcal{S R}\left[Q_{(J)}, \lambda_{(J)}\right]=\mathcal{S R}\left[Q_{(J)}, \lambda_{(J)}\right]$, which tells us that the ring is generated by degree 2 elements and the second part of (6.1) reduces the degree 2 elements to the same generators as $w \mathcal{S R}[Q, \lambda]$. See [BBCG15, Section 4] for details. However, in general, the cohomology ring $H^{*}(X ; \mathbb{Z})$ of a toric orbifold $X$ is not generated by degree 2 elements, hence the multiplication structure has plenty of divisibility because of singularities.

Recall that $X_{(J)}$ can be obtained from $X$ by a sequence of simplicial wedge constructions. Consider now the polytopal wedge $Q_{\left(J^{\prime}\right)}:=Q_{\left(F_{i}\right)}$ of the original simple lattice polytope $Q$ for some facet $F_{i}$ of $Q$.

We finish this paper by studying the vectors $\left\{z^{v^{\epsilon}} \mid v^{\epsilon} \in V\left(Q_{\left(J^{\prime}\right)}\right)\right\}$, with respect to the $\mathcal{R}$-characteristic pair $\left(Q_{\left(J^{\prime}\right)}, \lambda_{\left(J^{\prime}\right)}\right)$. Recall $V\left(Q_{\left(J^{\prime}\right)}\right)$ from (5.1). Theorem6.7 below tells us how to get

$$
z^{v^{\epsilon}}=\left(z_{0}^{v^{\epsilon}}, z_{1}^{v^{\epsilon}}, \ldots, z_{m}^{v^{\epsilon}}\right) \in \bigoplus_{m+1} \mathbb{Z}\left[u_{0}, u_{1}, \ldots, u_{n}\right]
$$

from $\left\{z^{v} \mid v \in V(Q)\right\}$, for each $v \in V(Q)$ and $\epsilon=+$ or - .

Remark 6.6. The polynomial ring $\mathbb{Z}\left[u_{0}, u_{1}, \ldots, u_{n}\right]$ stands for $H^{*}\left(B T^{n+1} ; \mathbb{Z}\right)$, where $T^{n+1}$ is the $(n+1)$-dimensional torus acting on $X_{\left(J^{\prime}\right)}$. The canonical embedding of $T^{n}$, the acting torus on $X(Q, \lambda)$, into the last $n$-coordinates of $T^{n+1}$ yields a canonical surjection $\mathbb{Z}\left[u_{0}, u_{1}, \ldots, u_{n}\right] \rightarrow \mathbb{Z}\left[u_{1}, \ldots, u_{n}\right]$. See Remark 6.1. 
According to (4.1) and (5.1), we have the following 3 types of vertices in $Q_{\left(J^{\prime}\right)}$;

$$
\begin{aligned}
& v_{i_{r}}^{-}=Q^{+} \cap Q^{-} \cap \bigcap_{a=1}^{n-1} F_{s_{a}\left(F_{i} \cap F_{s_{a}}\right)} \text { for some } v=F_{i} \cap \bigcap_{a=1}^{n-1} F_{s_{a}} \in V(Q), \\
& v^{+}=Q^{+} \cap \bigcap_{a=1}^{n}\left(F_{s_{a}} \times I\right) \text { for some } v=\bigcap_{a=1}^{n} F_{s_{a}} \text { and } v \notin V\left(F_{i}\right), \\
& v^{-}=Q^{-} \cap \bigcap_{a=1}^{n}\left(F_{s_{a}} \times I\right) \text { for some } v=\bigcap_{a=1}^{n} F_{s_{a}} \text { and } v \notin V\left(F_{i}\right) .
\end{aligned}
$$

We refer also to (5.2) and (5.3) for the indexing of facets in the above three cases.

Theorem 6.7. The weighted Stanley-Reisner ring of $\left(Q_{\left(J^{\prime}\right)}, \lambda_{\left(J^{\prime}\right)}\right)$ is related to that of $(Q, \lambda)$ as follows. For each vertex of one of the three types (6.2), (6.3) and (6.4) above, we have:

(1) $z^{v_{i_{r}}^{-}}=\left(u_{0}+z_{i}^{v_{i_{r}}}, z_{1}^{v_{i_{r}}}, \ldots, z_{m}^{v_{i_{r}}}\right)$ for $r=1, \ldots, k$;

(2) $z^{v^{+}}=\left(u_{0}, z_{1}^{v}, \ldots, z_{m}^{v}\right)$;

(3) $z^{v^{-}}=\left(0, z_{1}^{v^{-}}, \ldots, z_{m}^{v^{-}}\right)$, where

$$
z_{\ell}^{v^{-}}=\left\{\begin{array}{ll}
0 & \text { if } \ell \notin\left\{s_{1}, \ldots, s_{n}\right\} \\
\gamma_{\ell} u_{0}+z_{\ell}^{v} & \text { if } \ell \in\left\{s_{1}, \ldots, s_{n}\right\}
\end{array} \quad \text { and }\left[\begin{array}{c}
\gamma_{1} \\
\vdots \\
\gamma_{n}
\end{array}\right]=\Lambda_{v}^{-1} \cdot \lambda\left(F_{i}\right)^{t}\right.
$$

Proof. The proof follows from the direct computation of the inverse of $\Lambda_{\left(J^{\prime}\right)_{v^{\epsilon}}}$ for each $\epsilon=+$ and - . If $v$ is a vertex in $F_{i}$, i.e., $v=v_{i_{r}}$ for some $i_{r} \in\left\{i_{1}, \ldots, i_{k}\right\}$, and $v_{i_{r}}=F_{i} \cap F_{s_{1}} \cap \cdots \cap F_{s_{n-1}}$, then $\Lambda_{v}=\left[\lambda\left(F_{i}\right)^{t}\left|\lambda\left(F_{s_{1}}\right)^{t}\right| \cdots \mid \lambda\left(F_{s_{n-1}}\right)^{t}\right]$ and

$$
\Lambda_{\left(J^{\prime}\right) v_{i_{r}}^{-}}=\left[\begin{array}{ccccc}
1 & -1 & 0 & \cdots & 0 \\
0 & & & & \\
\vdots & \lambda\left(F_{i}\right)^{t} & \lambda\left(F_{s_{1}}\right)^{t} & \cdots & \lambda\left(F_{s_{n-1}}\right)^{t}
\end{array}\right]
$$

Its inverse is

$$
\Lambda_{\left(J^{\prime}\right)}{ }_{v_{i_{r}}^{-}}^{-1}=\left[\begin{array}{c|ccc}
1 & d_{1} & \cdots & d_{n} \\
\hline 0 & & & \\
\vdots & & \Lambda_{v}^{-1} & \\
0 & & &
\end{array}\right]
$$

where $\left(d_{1}, \ldots, d_{n}\right)$ is the first row of $\Lambda_{v}^{-1}$. Hence, by (i) and (ii) in page 18, we conclude (11).

Next, we assume that $v=F_{s_{1}} \cap \cdots \cap F_{s_{n}}$ with $i \notin\left\{s_{1}, \ldots, s_{n}\right\}$. The square matrices corresponding to $v^{+}=Q^{+} \cap \bigcap_{a=1}^{n}\left(F_{s_{a}} \times I\right)$ and $v^{-}=Q^{-} \cap \bigcap_{a=1}^{n}\left(F_{s_{a}} \times I\right)$ 
are

$$
\begin{aligned}
\Lambda_{\left(J^{\prime}\right) v^{+}} & =\left[\begin{array}{c|ccc}
1 & 0 & \cdots & 0 \\
\hline 0 & & & \\
\vdots & & \Lambda_{v} & \\
0 & & &
\end{array}\right]=\left[\begin{array}{ccccc}
1 & 0 & \cdots & 0 \\
0 & & & \\
\vdots & \lambda\left(F_{s_{1}}\right)^{t} & \cdots & \lambda\left(F_{s_{n}}\right)^{t} \\
0 & & &
\end{array}\right], \\
\Lambda_{\left(J^{\prime}\right)_{v^{-}}} & =\left[\begin{array}{c|ccc}
1 & 0 & \cdots & 0 \\
\hline \lambda\left(F_{i}\right)^{t} & & & \\
& & &
\end{array}\right]=\left[\begin{array}{cccc}
-1 & 0 & \ldots & 0 \\
\lambda\left(F_{i}\right)^{t} & \lambda\left(F_{s_{1}}\right)^{t} & \ldots & \lambda\left(F_{s_{n}}\right)^{t}
\end{array}\right] .
\end{aligned}
$$

respectively. Their inverses are

$$
\Lambda_{\left(J^{\prime}\right)_{v^{+}}^{-1}}=\left[\begin{array}{c|ccc}
1 & 0 & \cdots & 0 \\
\hline 0 & & \\
\vdots & & \Lambda_{v}^{-1} & \\
0 & &
\end{array}\right]
$$

and

$$
\Lambda_{\left(J^{\prime}\right)_{v^{-}}^{-1}}=\left[\begin{array}{c|ccc}
-1 & 0 & \cdots & 0 \\
\hline \gamma_{1} & & & \\
\vdots & & \Lambda_{v}^{-1} & \\
\gamma_{n} & &
\end{array}\right] \text {, where }\left[\begin{array}{c}
\gamma_{1} \\
\vdots \\
\gamma_{n}
\end{array}\right]=\Lambda_{v}^{-1} \cdot \lambda\left(F_{i}\right)^{t}
$$

Now, the results for (2) and (3) are straightforward from (6.5) and (6.6), respectively.

\section{REFERENCES}

[AM69] M. F. Atiyah and I. G. Macdonald, Introduction to commutative algebra, AddisonWesley Publishing Co., Reading, Mass.-London-Don Mills, Ont., 1969. MR 0242802

[BBCG] A. Bahri, M. Bendersky, F. R. Cohen, and S. Gitler, A generalization of the davisjanuszkiewicz construction and applications to toric manifolds and iterated polyhedral products, To appear in: Perspectives in Lie Theory, Springer. Online at: http://arxiv.org/abs/1311.4256

[BBCG15] - Operations on polyhedral products and a new topological construction of infinite families of toric manifolds, Homology Homotopy Appl. 17 (2015), no. 2, 137-160. MR 3426378

[BFNR13] Anthony Bahri, Matthias Franz, Dietrich Notbohm, and Nigel Ray, The classification of weighted projective spaces, Fund. Math. 220 (2013), no. 3, 217-226. MR 3040671

[BFR09] Anthony Bahri, Matthias Franz, and Nigel Ray, The equivariant cohomology ring of weighted projective space, Math. Proc. Cambridge Philos. Soc. 146 (2009), no. 2, 395-405. MR 2475973 (2010a:57054)

[BNSS] Anthony Bahri, Dietrich Notbohm, Soumen Sarkar, and Jongbaek Song, On integral cohomology of certain orbifolds, arXiv:1711.01748.

[BP15] Victor M. Buchstaber and Taras E. Panov, Toric topology, Mathematical Surveys and Monographs, vol. 204, American Mathematical Society, Providence, RI, 2015. MR 3363157

[BSS17] Anthony Bahri, Soumen Sarkar, and Jongbaek Song, On the integral cohomology ring of toric orbifolds and singular toric varieties, Algebr. Geom. Topol. 17 (2017), no. 6, 3779-3810. MR 3709660

[CLS11] David A. Cox, John B. Little, and Henry K. Schenck, Toric varieties, Graduate Studies in Mathematics, vol. 124, American Mathematical Society, Providence, RI, 2011. MR 2810322 (2012g:14094) 
[CP16] Suyoung Choi and Hanchul Park, Wedge operations and torus symmetries, Tohoku Math. J. (2) 68 (2016), no. 1, 91-138. MR 3476138

[CP17] W Wedge Operations and Torus Symmetries II, Canad. J. Math. 69 (2017), no. 4, 767-789. MR 3679694

[DJ91] Michael W. Davis and Tadeusz Januszkiewicz, Convex polytopes, Coxeter orbifolds and torus actions, Duke Math. J. 62 (1991), no. 2, 417-451. MR 1104531 (92i:52012)

[Ewa86] Günter Ewald, Spherical complexes and nonprojective toric varieties, Discrete Comput. Geom. 1 (1986), no. 2, 115-122. MR 834053

[Fis92] Stephan Fischli, On toric varieties, Ph.D. thesis, Universität Bern (1992).

[FP07] Matthias Franz and Volker Puppe, Exact cohomology sequences with integral coefficients for torus actions, Transform. Groups 12 (2007), no. 1, 65-76. MR 2308029

[Ful93] William Fulton, Introduction to toric varieties, Annals of Mathematics Studies, vol. 131, Princeton University Press, Princeton, NJ, 1993, The William H. Roever Lectures in Geometry. MR 1234037 (94g:14028)

[GKM98] Mark Goresky, Robert Kottwitz, and Robert MacPherson, Equivariant cohomology, Koszul duality, and the localization theorem, Invent. Math. 131 (1998), no. 1, 25-83. MR 1489894

[Ill78] Sören Illman, Smooth equivariant triangulations of $G$-manifolds for $G$ a finite group, Math. Ann. 233 (1978), no. 3, 199-220. MR 0500993 (58 \#18474)

[Jor98] Arno Jordan, Homology and cohomology of toric varieties, Ph.D. thesis, University of Konstanz (1998).

[Kaw73] Tetsuro Kawasaki, Cohomology of twisted projective spaces and lens complexes, Math. Ann. 206 (1973), 243-248. MR 0339247

[KMZ17] Hideya Kuwata, Mikiya Masuda, and Haozhi Zeng, Torsion in the cohomology of torus orbifolds, Chin. Ann. Math. Ser. B 38 (2017), no. 6, 1247-1268. MR 3721698

[LdM89] Santiago López de Medrano, Topology of the intersection of quadrics in $\mathbf{R}^{n}$, Algebraic topology (Arcata, CA, 1986), Lecture Notes in Math., vol. 1370, Springer, Berlin, 1989, pp. 280-292. MR 1000384

[PB80] J. Scott Provan and Louis J. Billera, Decompositions of simplicial complexes related to diameters of convex polyhedra, Math. Oper. Res. 5 (1980), no. 4, 576-594. MR 593648 (82c:52010)

[PS10] Mainak Poddar and Soumen Sarkar, On quasitoric orbifolds, Osaka J. Math. 47 (2010), no. 4, 1055-1076. MR 2791564 (2012e:57058)

[Zie95] Günter M. Ziegler, Lectures on polytopes, Graduate Texts in Mathematics, vol. 152, Springer-Verlag, New York, 1995. MR 1311028 (96a:52011)

Department of Mathematics, Rider University, NJ, USA

E-mail address: bahri@rider.edu

Department of Mathematics, Indian Institute of Technology Madras, Chennai, India

E-mail address: soumensarkar20@gmail.com

Department of Mathematical Sciences, Kaist, Daejeon, Republic of Korea

E-mail address: jongbaek.song@gmail.com 